\title{
Vibronic interaction in trans- dichloroethene studied by vibration- and angle-resolved photoelectron spectroscopy using 19-90 eV photon energy
}

Ayse T. Duran ${ }^{(a, b)}$, Ivan Powis *(a) ${ }^{\left({ }^{2}\right.}$ David M.P. Holland ${ }^{(c)}$, Christophe Nicolas ${ }^{(d)}$, John Bozek ${ }^{(d)}$, A. B. Trofimov ${ }^{(e, f)}$, E. K. Grigoricheva ${ }^{(e)}$, A. D. Skitnevskaya ${ }^{(e)}$

a) School of Chemistry, The University of Nottingham, University Park, Nottingham NG7 2RD, UK

b) Photek Ltd, Castleham Road, St Leonards on Sea, East Sussex TN38 9NS, UK

${ }^{c)}$ Daresbury Laboratory, Daresbury, Warrington, Cheshire WA4 4AD, UK

d) Synchrotron SOLEIL, I'Orme des Merisiers, Saint-Aubin, BP 48, 91192 Gif-sur-Yvette, France

e) Laboratory of Quantum Chemical Modelling of Molecular Systems, Irkutsk State University, Karl Marx Str. 1, 664003 Irkutsk, Russia

f) Favorsky's Institute of Chemistry, SB RAS, Favorsky Str. 1, 664033 Irkutsk, Russia

*Corresponding Author:

Email: ivan.powis@nottingham.ac.uk

Phone: +44 1159513467

Keywords: Photoelectron Spectroscopy; Angular Distribution; Franck-Condon; Vibronic Interaction 


\begin{abstract}
Valence photoelectron spectra and photoelectron angular distributions of trans-dichloroethene have been measured with vibrational resolution at photon energies between 19-90 eV. Calculations of photoelectron anisotropy parameters, $\beta$, and harmonic vibrational modes help provide initial insight into the molecular structure. The photon energy range encompasses the expected position of the atomic $\mathrm{Cl} 3 p$ Cooper minimum. A corresponding dip observed here in the anisotropy of certain photoelectron bands permits the identification and characterisation of those molecular orbitals that retain a localised atomic $\mathrm{Cl}$ character.

The adiabatic approximation holds for the $X^{2} A_{u}$ state photoelectron band, but vibronic coupling was inferred within the $A-B-C$ and the $D-E$ states by noting various failures of the Franck-Condon model, including vibrationally dependent $\beta$-parameters. This is further explored using the linear vibronic coupling model with interaction parameters obtained from $a b$ initio calculations. The $A / B$ photoelectron band is appreciably affected by vibronic coupling owing to the low-lying conical intersection of the $A^{2} A_{g}$ and $B{ }^{2} B_{u}$ states. The $C^{2} B_{g}$ band is also affected, but to a lesser extent. The adiabatic minima of the $D^{2} A_{u}$ and $E{ }^{2} A_{g}$ states are almost degenerate and the vibronic interaction between these states is considerable. The potential energy surface of the $D^{2} A_{u}$ state is predicted to have a double-minimum shape with respect to the $a_{\mathrm{u}}$ deformations of the molecular structure. The irregular vibrational structure of the resulting single photoelectron band reflects the non-adiabatic nuclear dynamics occurring on the two coupled potential energy surfaces above the energy of their conical intersection.
\end{abstract}




\section{Introduction}

Molecular photoionization is often considered within a model employing the Born-Oppenheimer and Franck-Condon approximations, ${ }^{1}$ wherein each electronic state has an associated isolated potential energy surface. Under these conditions, the photoelectron spectrum due to a particular electronic state exhibits regular progressions involving only the totally symmetrical vibrational modes. However, this simple picture may be changed radically by vibronic interaction between neighbouring ionic states that modifies the photoionization dynamics. The interaction of two or more energetically close-lying electronic states, through the nuclear motion, results in a breakdown of the Born-Oppenheimer approximation and the ensuing non-adiabatic effects, which account for motion of the nuclei on more than one potential energy surface, are evident in the photoelectron band vibrational structure. Observable changes can vary between slightly irregular (both in terms of vibrational energies and intensities) but still recognizable progressions in the case of weak vibronic coupling, to a complete loss of any regular vibrational pattern for strong vibronic coupling. In the latter case, vibronic coupling calculations predict numerous vibrational excitations, some of which involve non-totally symmetrical vibrational modes. The summation of these excitations may result in a diffuse photoelectron band with no identifiable structure.

Although less frequently studied in this context, the vibrationally resolved photoelectron anisotropy parameter, $\beta$, may provide further detailed evidence of vibronic interaction between states. ${ }^{2}$ In a recent investigation of cis-dichloroethene ${ }^{3,4}$ it was shown that vibronic coupling strongly influences the $A / B / C$ states, and the $D / E$ states. These effects not only modify the vibrational structure but also introduce unexpected energy dependencies in the photoelectron angular distribution (PAD). In the isolated electronic state Franck-Condon approximation, the motions of the electrons and nuclei are assumed to be fully decoupled, and $\beta$-parameters are expected to be independent of vibrational state. Conversely, variations of the PAD measured across a photoelectron band, even when the vibrational structure of the band is quasi-regular, can be a sensitive indicator of vibronic interaction. This behaviour has been reported in the $D / E$ state interaction in cis-dichloroethene, ${ }^{3}$ and has also been seen in a recent similar study of oxalyl chloride. ${ }^{5}$ Even more strikingly, in the presence of a strong vibronic interaction producing a diffuse band structure, such as the $A / B$ state region of cisdichloroethene, the PAD and its $\beta$-parameter can transition between two limits, reflecting the changing electronic character in the coupled states. ${ }^{2,3,4}$

In situations where the Born-Oppenheimer and Franck-Condon approximations break down, an adequate theoretical modelling accounting for vibronic coupling of the electronic states involved is of crucial importance for the interpretation of the observations. At present, the non-adiabatic effects can be efficiently treated within the framework of the general vibronic coupling theory, ${ }^{1,6,7}$ which is also very well suited for radical cationic states interacting via totally and non-totally symmetric vibrational modes. ${ }^{8-13}$ The approach is based on the model Hamiltonian theory for diabatic electronic states and in its simplest formulation employs a linear approximation for the potential energy terms. ${ }^{1,6}$ The resulting linear vibronic coupling (LVC) models can be parameterized using results of ab initio electronic structure calculations and employed for studies of nuclear dynamics.

In this paper, we extend our earlier vibration and angle resolved studies of cis-dichloroethene $e^{3,4}$ to investigate the trans isomer of this compound. Because the strength of vibronic interactions depends upon both the energetic separation between the participating ionic states and on the molecular geometry (through the allowed totally symmetrical and the vibronically induced non-totally 
symmetrical vibrational modes) we expect the interstate mixing in the trans isomer to differ from that already observed in the cis isomer. These differences can be attributed to shifts in the valence orbital binding energies ${ }^{14}$ and to different point group symmetries $\left(C_{2 v}\right.$ for the cis isomer, $C_{2 h}$ for the trans isomer).

Previous studies of the photoionization and electronic structure of trans-dichloroethene include photoelectron spectra recorded with $\mathrm{Hel}^{15-19} \mathrm{Hell}^{18,20}{ }^{18 \mathrm{~K} \alpha}{ }^{21}$ and synchrotron ${ }^{22}$ radiation. A more recent comparative study of dichloroethene isomers employed threshold photoelectron spectroscopy (TPES) and synchrotron radiation. ${ }^{14}$ The valence orbitals of trans-dichloroethene have also been studied using symmetric noncoplanar $(e, 2 e)$ spectroscopy. ${ }^{23}$ These experimental investigations provided interpretations that were advanced by calculation of vertical ionization energies, and subsequent theoretical work has also addressed the calculation of adiabatic ionization energies. ${ }^{24,25}$ The broad features observed in the inner valence region of trans-dichloroethene, due to electron correlation, have been studied theoretically. ${ }^{20,26}$ The currently accepted valence electron orbital ordering is ...(7ag $)^{2}\left(7 b_{u}\right)^{2}\left(8 a_{g}\right)^{2}\left(8 b_{u}\right)^{2}\left(9 a_{g}\right)^{2}\left(2 a_{u}\right)^{2}\left(2 b_{g}\right)^{2}\left(9 b_{u}\right)^{2}\left(10 a_{g}\right)^{2}\left(3 a_{u}\right)^{2}$ although density functional calculations may reverse the $8 \mathrm{~b}_{\mathrm{u}} / 8 \mathrm{a}_{\mathrm{g}}$ ordering ${ }^{27}$ and other discrepancies are found in the older literature that may be attributed to the limitations of computational methods available at the time.

Much higher resolution experimental spectra, although restricted to the electronic ground state of the cation, have been recorded using VUV-laser pulsed field ionization with photoelectron (PFI-PES) ${ }^{28}$ and mass-analysed threshold ion (PFI-MATI) ${ }^{29}$ detection. Vibrational assignments were achieved in either case using high level frequency calculations and Franck-Condon intensity simulations. This approach was also adopted in an earlier theoretical study of the cis-, trans-, and 1,1-dichloroethene isomers to predict vibrational structure of not only their ground state cations but also of the next three excited states. ${ }^{25}$ Some further insights into vibrational structure of the trans-dichloroethene cation may be obtained from a vibrationally resolved VUV absorption study of the associated Rydberg states (sharing a common molecular core with the free cation). ${ }^{27}$

In the current investigation, measurements of the valence photoelectron spectrum and PADs of transdichloroethene are repeated using a number of photon energies between 19 and $90 \mathrm{eV}$. This extended photon energy range offers possible assistance with confirming or discounting accidentally encountered continuum resonances, since these may also influence the PAD. It has also long been recognised that the $\sigma$ or $\pi$ orbital character may be inferred from their characteristic energydependent $\beta$-parameters in the range from threshold to several tens eV. ${ }^{30,31}$ More significantly, this range encompasses the expected position of the atomic $\mathrm{Cl} 3 p$ Cooper minimum (CM). ${ }^{32-34}$ The influence of a $\mathrm{CM}$ is usually best evidenced in the photoelectron angular distributions, and such observations will allow for close examination of the associated photoionization dynamics. Breakdown of the Franck-Condon approximation in molecular systems has been predicted to be induced by CM phenomena, ${ }^{35}$ and some weak evidence of vibrationally dependent $\beta$-parameters has been adduced in the vicinity of the atomic-like bromine $\mathrm{CM}$ in the photoionization of bromobenzene. ${ }^{36}$ More confidently, we can expect that any observation of CM in the PADs of small molecular halides will reveal the extent of halogen lone-pair character in the molecular structure, as was already demonstrated for several other molecular chlorides. $3,30,37-40$

In this work, photoelectron spectra are recorded as pairs using synchrotron radiation with linear polarization set, alternately, to align perpendicular and parallel to the detection axis accepted by the spectrometer. The general form of the photoelectron angular distribution is, as well known, given by ${ }^{41}$ 


$$
I(\theta)=1+\beta P_{2}(\cos \theta),
$$

where $P_{2}$ is the second Legendre polynomial, and $\theta$ is the angle of electron emission with respect to the polarization vector and so the perpendicular- and parallel-polarized intensities may be denoted as $I\left(90^{\circ}\right)$ and $I\left(0^{\circ}\right)$, respectively. These spectra are conveniently combined in two different ways. The first synthesises a so-called Magic Angle spectrum, IMA, which shows no dependence on, and so requires no knowledge of, the $\beta$-parameter. This is, of course, advantageous for initial examination of vibrational intensities. $I_{\mathrm{MA}}$ is conventionally obtained as an independently recorded spectrum at $\theta=$ $54.7^{\circ}$, since at this angle the $2^{\text {nd }}$ Legendre polynomial term (Eq. (1)) vanishes. Using the properties of the Legendre polynomials one can, however, show a similar cancellation of angular dependence by forming

$$
I_{M A}\left(\equiv I\left(54.7^{\circ}\right)\right)=I\left(0^{\circ}\right)+2 I\left(90^{\circ}\right) .
$$

Alternatively, with a little more manipulation, one finds a second useful combination giving a value for $\beta$ :

$$
\beta=2\left(I\left(0^{\circ}\right)-I\left(90^{\circ}\right)\right) /\left(I\left(0^{\circ}\right)+2 I\left(90^{\circ}\right)\right)
$$

In what follows, we first outline the experimental and computational methods. A full inner and outer valence region spectrum is then briefly discussed, after which we focus on the outer valence region having binding energies below $18 \mathrm{eV}$. The $\beta$-parameters and branching ratios (proportional to the relative cross-sections) are first presented and compared with theoretical calculations on a band by band basis, considering just electronic state dependency. Subsequently, the vibrational structure (intensity and angular distribution) is analysed, initially in the context of Born-Oppenheimer assumptions and using Franck-Condon simulations. Finally, computational modelling of the vibronic interactions that are revealed in this molecule is presented and discussed.

\section{Methods}

The current trans-dichloroethene investigation closely parallels the experimental and computational methods of our earlier investigation of cis-dichloroethene ${ }^{3,4}$ and so here we provide only brief details; the reader is referred to these earlier papers for a fuller account.

\section{A. Experimental}

The polarization dependent photoelectron spectra were recorded with a VG Scienta R4000 hemispherical electron analyser mounted on the soft X-ray undulator-based PLÉIADES beamline at the SOLEIL synchrotron facility. The monochromator exit slit width was set at $50 \mu \mathrm{m}$, which, when combined with the 400 grooves $/ \mathrm{mm}$ grating, corresponds to theoretical optical resolutions of $\sim 1$ and $7 \mathrm{meV}$, respectively, at photon energies of 19 and $90 \mathrm{eV}$. The photoelectron spectra were measured using an analyser pass energy of $10 \mathrm{eV}$ and a $0.2 \mathrm{~mm}$ curved entrance slit, resulting in a theoretical spectrometer resolution of $5 \mathrm{meV}$. Translational Doppler broadening also contributes to the overall peak width. ${ }^{42}$ Such contributions amount to $\sim 3.9$ and $11.4 \mathrm{eV}$ for electrons ejected from transdichloroethene with kinetic energies of 9.369 and 80.369 (corresponding to the formation of the $X^{2} A_{u}$ state in the vibrationally unexcited level using photon energies of 19 and $90 \mathrm{eV}$, respectively). 
Trans-dichloroethene is a liquid with a significant vapour pressure at room temperature. After several freeze/pump/thaw cycles to remove volatile impurities, the vapour was introduced into the experimental chamber without heating.

All the spectra were normalized to the sample pressure, the accumulation time, and the photon flux prior to processing. The transmission efficiency of the electron analyser was determined, as described by Jauhiainen et al, ${ }^{43}$ and all the spectra were corrected for this variation. These normalized spectra were then used in conjunction with eqs (1) and (3) to evaluate the experimental photoelectron anisotropy parameters and branching ratios associated with a particular binding energy range, as described previously. ${ }^{5,44}$ Table 1 lists the binding energy ranges used to analyse the photoelectron spectra of trans-dichloroethene. The mean $\beta$-parameters and branching ratios evaluated in this manner are thus vibrationally averaged values and can be compared with corresponding theoretical predictions obtained in fixed nuclei calculations. Our analysis procedure also allows the variation in the $\beta$-parameter as a function of the binding energy across a specific photoelectron band to be determined, thereby enabling the variation in the $\beta$-value associated with specific vibrational excitations to be observed. Errors for the $\beta$-values were estimated by propagation of the assumed statistical (Poisson) counting uncertainty through the evaluation of eq (3). The resulting error bars do not include any adjustment for possible systematic error that may arise, for example, from non-ideal spectrometer performance.

The electron binding energy scale was calibrated by comparing the position of the adiabatic ionization transition of the HOMO (whose identification is discussed in the section on Vibrationally Resolved Results of the $X$-band and in Supplementary Material) with the value of $9.631 \mathrm{eV}$ obtained in the PFI$\mathrm{PES}^{28}$ and PFI-MATI ${ }^{29}$ studies.

\section{B. Computational}

The vertical ionization spectra have been computed using the outer valence Green's function (OVGF) method, ${ }^{45,46}$ the equation-of-motion coupled cluster (EOM-IP-CCSD) approach, ${ }^{47-49}$ and the third-order algebraic-diagrammatic construction $(A D C(3))$ approximation for the one-particle Green's function. ${ }^{45,50,51}$ The computational schemes are implemented respectively in the GAUSSIAN (versions $09,16),{ }^{52} \mathrm{Q}$-Chem (v4.2), ${ }^{53}$ and local version of GAMESS (2014 R1) ${ }^{54,55}$ program packages. In the calculations of ionization spectra, the carbon and chlorine $\mathrm{K}$-shell orbitals and the chlorine L-shell orbitals were kept frozen. Theoretical photoelectron spectra were constructed from the $A D C(3)$ results by convoluting the calculated data with Lorentzians of $0.4 \mathrm{eV}$ (FWHM). All these electronic structure calculations were performed using the cc-pVTZ basis set ${ }^{56,57}$ and an optimised MP2 groundstate geometry. This planar $\left(C_{2 h}\right)$ geometry corresponds to experimental gas-phase electron diffraction measurements ${ }^{58}$ and coordinate values are given in Table S1 (Supplementary Material).

Photoionization properties (cross-sections, $\beta$-parameters) were calculated at the same fixed, initial geometry using a static-exchange, independent electron, continuum multiple scattering model ${ }^{59,60}$ with a $\mathrm{X} \alpha$ exchange potential (CMS-X $\alpha$ ). Our method has been described previously, ${ }^{61,62}$ and is only briefly summarized here. A self-consistent neutral molecule potential, modelled as overlapping spherical regions centred on each atomic site, is constructed with the whole enclosed within a spherically symmetric outer sphere that extends to infinity. Within these regions the exchange contribution to an effective one-electron potential is represented using the Slater Xa local density approximation. ${ }^{63}$ The wavefunctions are expressed in a symmetry-adapted basis of spherical harmonic functions on each centre, with radial functions obtained by direct numerical integration within the 
spherical zones of the potential. After adaptation to ensure the correct asymptotic Columbic behaviour expected for the continuum electron-ion system, one electron continuum functions are found by solving the scattering problem with this potential. Electric dipole photoionization matrix elements, and hence cross-sections and $\beta$-parameters, may then be calculated. The parameters used for construction of the scattering potential and the angular basis are given in Table S2 (Supplementary Material).

Franck-Condon simulations were prepared using harmonic vibrational frequencies and normal modes. For the excited cation state calculations, we used either coupled cluster methods with the equationsof-motion formalism (EOM-CCSD) and the cc-pVTZ basis, or time-dependent density functional theory with the B3LYP functional (TD-B3LYP) and 6-311G(d,p) basis, both implemented in the Gaussian 16 suite of programs. ${ }^{52}$ Franck-Condon factors were then calculated using the adiabatic hessian model, including Duschinsky rotations, provided in Gaussian 16. ${ }^{52}$ With the EOM-CCSD/cc-pVTZ excited state parameters we used neutral ground state normal modes obtained at the MP2/cc-pVTZ level, while for the TD-DFT based simulations we switched, for consistency, to a B3LYP/6-311G(d,p) calculation.

The vibronic coupling calculations were performed for two groups of excited states of the transdichloroethene radical cation. The states belonging to each group, $A^{2} \mathrm{~A}_{\mathrm{g}}-B^{2} \mathrm{~B}_{\mathrm{u}}-C^{2} \mathrm{~B}_{\mathrm{g}}$ and $D^{2} \mathrm{~A}_{u}-E^{2} \mathrm{~A}_{\mathrm{g}}$, are close in energy and can interact vibronically. At the same time, since the groups are separated by an appreciable energy interval, they were treated separately.

In each group, all possible vibronic interactions were taken into account at the level of the LVC approximation. In the $A-B-C$ group, these are the interactions via the $b_{\mathrm{u}}, a_{\mathrm{u}}$ and $b_{\mathrm{g}}$ non-totally symmetric modes $\left(\mathrm{A}_{\mathrm{g}} \times \mathrm{B}_{\mathrm{u}} \times b_{\mathrm{u}} \supset \mathrm{A}_{\mathrm{g}}, \mathrm{B}_{\mathrm{u}} \times \mathrm{B}_{\mathrm{g}} \times a_{\mathrm{u}} \supset \mathrm{A}_{\mathrm{g}}, \mathrm{A}_{\mathrm{g}} \times \mathrm{B}_{\mathrm{g}} \times b_{\mathrm{g}} \supset \mathrm{A}_{\mathrm{g}}\right)$, and in the $D-E$ group, this is the interaction via the $a_{\mathrm{u}}$ modes $\left(\mathrm{A}_{\mathrm{u}} \times \mathrm{A}_{\mathrm{g}} \times a_{\mathrm{u}} \supset \mathrm{A}_{\mathrm{g}}\right)$. Two appropriate vibronic coupling models were formulated within the framework of a general vibronic coupling theory and used for nuclear dynamics calculations. ${ }^{1}$ The actual computational protocol closely follows that of our previous work on the cisisomer. ${ }^{4}$ For this reason, here we only outline the approach being used and introduce the quantities appearing later in the discussion of the results.

In the present LVC treatment, each group of coupled cationic states was described by a matrix model Hamiltonian $\hat{\mathbf{H}}$ which is defined as follows: ${ }^{1}$

$$
(\hat{\mathbf{H}})_{i j}=\left\{\begin{array}{c}
\hat{H}_{0}+E_{i}+\sqrt{2} \sum_{s \in \gamma} \kappa_{i}^{s} Q_{s}, \quad i=j \\
\sqrt{2} \sum_{s \in \mathcal{V}} \lambda_{i j}^{s} Q_{s}, \quad i \neq j
\end{array}\right.
$$

Here $E_{i}$ denote vertical ionization energies associated with the $i$-th cationic state, $\kappa_{i}^{s}$ and $\lambda_{i j}^{s}$ are the so-called intrastate and interstate coupling constants, respectively, and $Q_{s}$ are the dimensionless normal coordinates associated with the totally symmetric $(\gamma)$ and non-totally symmetric $(v)$ normal modes $v_{\mathrm{s}}$. The Hamiltonian $\hat{H}_{0}$ refers to the electronic ground state and describes the set of noninteracting harmonic oscillators, $v_{s}$, with frequencies $\omega_{s}$.

The vibronic transition energies, as well as the corresponding spectral intensities, are computed variationally. For this purpose, the Hamiltonian of Eq. (4) is expressed in a direct-product harmonic 
oscillator basis of $\hat{H}_{0}$ eigenstates, and a computational procedure based on the Lanczos algorithm is employed. ${ }^{1}$ The spectral envelope was obtained by convoluting the calculated spectrum with Lorentzians of 0.011 eV FWHM which accounted for the phenomenological broadening.

The vibronic interaction in the Hamiltonian (Eq. (4)) can be "switched off" by setting all $\lambda_{i j}^{s}$ constants to zero. The resulting model allows the spectrum to be evaluated analytically and yields intensities that follow a Poisson distribution. Comparison of such a Poisson spectrum (without vibronic coupling) with the vibronic spectrum provides useful insight into the role of vibronic coupling.

In order to set up the model of Eq. (4), the parameters $E_{i}, \omega_{s}, \kappa_{i}^{s}, \lambda_{i j}^{s}$ have to be known. The groundstate vibrational frequencies $\omega_{s}$ were again those computed at the MP2/cc-pVTZ level of theory (Table S3) as used for the full FC simulations. The vertical ionization energies $E_{i}$, as well as the coupling constants $\kappa_{i}^{S}$ and $\lambda_{i j}^{S}$, were obtained using the OVGF/cc-pVTZ calculations. In the determination of the coupling constants, the OVGF calculations were performed for molecular structures distorted along the normal coordinates of the respective vibrational modes. The potential energy part of the LVC model Hamiltonian (Eq. (4)) was then fitted to the results of the OVGF calculations using equations presented in our previous paper. ${ }^{4}$

For further use we introduce the Poisson parameters $a_{i s}=\left(\kappa_{i}^{s} / \omega_{s}\right)^{2}$ and $a_{i j s}=\left(\lambda_{i j}^{s} / \omega_{s}\right)^{2}$ for the totally and non-totally symmetric modes, respectively. ${ }^{1}$ These dimensionless quantities reflect the extent to which the respective mode is excited.

\section{Results and Discussion}

\section{A. Overview of the complete valence shell photoelectron spectrum}

Calculated vertical ionization energies are compared with experimental values in Table 2, and the correlated OVGF, EOM-IP-CCSD, and ADC(3) methods are seen to display good agreement with experiment and with each other. The EOM-IP-CCSD and ADC(3) methods are in many respects similar computational schemes. Both treat $2 \mathrm{~h}-1 \mathrm{p}$ (two-hole one-particle) states at the level of first order perturbation theory. But while $\mathrm{ADC}(3)$ provides a consistent third-order description of the ionization energies and relative intensities (pole strengths) of $1 \mathrm{~h}$ (one-hole) transitions, in EOM-IP-CCSD these quantities are treated consistently only through second and first-order perturbation theory, respectively. We therefore prefer the $A D C(3)$ results for simulating the electronic ionization spectra as has proved successful in previous studies. ${ }^{3,4,30,31,37-39}$

In Figure 1 we compare the theoretical spectrum obtained from the ADC(3)/cc-pVTZ energies and pole strengths with the experimental photoelectron spectrum of trans-dichloroethene, recorded at a photon energy of $80 \mathrm{eV}$ using parallel polarized radiation. The overall agreement between the experimental and calculated spectra is good, thereby allowing the observed structure to be identified. The calculated pole strengths shown in Table $2(\sim 0.9)$ indicate that the single particle model of ionization, ${ }^{64}$ where ionization from a specific orbital gives rise to a single photoelectron band, is valid for the eight least tightly bound orbitals in trans-dichloroethene. 
The single particle model, however, begins to breakdown for the $7 b_{u}$ orbital, and several satellite states are predicted to occur at binding energies both below and above that for the main-line (Figure 1). In the experimental spectrum, the photoelectron band due to the $7 \mathrm{~b}_{\mathrm{u}}$ orbital displays three distinct peaks, at binding energies of 19.01, 19.31 and $19.52 \mathrm{eV}$. The experimental spectrum also exhibits a general rise in the background intensity, beginning at $17.6 \mathrm{eV}$, and this seems to match the location of the satellites predicted for the $8 \mathrm{a}_{\mathrm{g}}$ orbital. The prominent peak centred at a binding energy of 22.45 $\mathrm{eV}$ appears to correlate with the cluster of satellites associated with the $7 \mathrm{a}_{\mathrm{g}}$ orbital, and, to higher energy, the numerous low intensity satellites predicted for the $6 b_{u}$ and $6 a_{g}$ orbitals probably account for the broad features in the experimental spectrum.

According to the $A D C(3)$ calculations, a shake-up satellite involving the ejection of one electron from the $3 a_{u}$ orbital, together with the excitation of another electron from the same orbital into a low lying $b_{g}\left(\pi^{*}\right)$ valence orbital, occurs at a binding energy of $14.45 \mathrm{eV}$, which would signal an incipient failure of the single particle model. However, this predicted shake-up state, which lies just above the $D / E$ bands due to ionization from the $2 \mathrm{a}_{\mathrm{u}}$ and $9 \mathrm{a}_{\mathrm{g}}$ orbitals, does not appear to give rise to a distinct feature in the experimental spectrum.

\section{B. State resolved results}

Henceforward, we focus our attention on the outer valence region below a binding energy of $18 \mathrm{eV}$, and especially the six bands below $15 \mathrm{eV}$. Figure 2 shows the Magic Angle spectrum recorded at $h v=$ $22.0 \mathrm{eV}$. A basic estimate of the vertical ionization energies obtained from Hartree-Fock (HF) orbital energies using Koopmans' theorem is placed alongside more sophisticated methods (OVGF, ADC(3) and EOM-IP-CCSD) in Table 2, where all but the HF/Koopmans' values are seen to closely match the experimental peak positions. The inclusion of the OVGF results in Fig. 2 illustrates the good correspondence achieved by these calculations. Assignments can thus be made for at least the first six photoelectron bands, based upon the orbital identifications given in Table 2, and are shown in Fig. 2. A visual representation of these orbitals is provided in Figure 3, and Mulliken orbital populations are listed in Table 3.

The experimental photoelectron anisotropy parameters, $\beta$, averaged across the individual electronic bands (defined by their binding energy ranges given in Table 1), are shown for each photon energy examined in our study in Figure 4. The $\beta$-parameter for the HOMO has the typical characteristics associated with a $\pi$ orbital: an essentially monotonic but rapid rise from a low value near threshold, reaching a plateau with a limiting $\beta \approx 1.5$ at high kinetic energies. The experimental $\beta$-parameters for the next four bands, $A-D$, all show a distinct dip at a photon energy $\sim 45 \mathrm{eV}$, coinciding with the position of the atomic $\mathrm{Cl} 3 p$ Cooper minimum. ${ }^{33}$ This dip is well reproduced in the theoretical $\beta$ curves calculated for the corresponding orbital ionizations, helping to corroborate the identification of the observed dips as Cooper minima. Examining the iso-surface density plots for the $10 \mathrm{a}_{\mathrm{g}}, 9 \mathrm{~b}_{\mathrm{u}}$, and $2 \mathrm{~b}_{\mathrm{g}}$ orbitals (Fig. 3 and Table 3), associated with the $A, B$ and $C$ electronic states, it is seen that these orbitals possess a very high $\mathrm{Cl}$ lone pair character and so it is expected that they would show strong atomic-like behaviour with pronounced Cooper minima. ${ }^{34}$ The fourth of these orbitals, $2 \mathrm{a}_{\mathrm{u}}$, associated with the $D$ state, has a significantly reduced $\mathrm{Cl}$ population (0.58), and the out-of-plane symmetry of the $\mathrm{Cl}$ lone pair orbitals here permits interaction with the $\mathrm{C}=\mathrm{C} \pi$ bonding, consequently reducing the atomic-like character. This reduction is reflected in a shallower Cooper minimum, seen in both the experimental and theoretical $D$ state $\beta$-parameters. 
The $E$ band (strictly the high energy side of the strongly overlapped $D / E$ bands) shows an overall reduction in the $\beta$-parameter across the photon energy range, but also provides the greatest discrepancy with its corresponding $9 \mathrm{ag}^{-1}$ calculation, although both theory and experiment still display a shallow Cooper minimum. The Cooper minimum aside, the predicted $9 \mathrm{ag}^{-1}$ behaviour differs from that of the preceding $\pi$ orbitals, for example the HOMO, by rising much more slowly from the low value at threshold. This is a known behaviour typical of $\sigma$-type molecular orbitals, and could hence be expected from the $9 \mathrm{a}_{\mathrm{g}}$ orbital character (Fig. 3 and Table 3). One explanation for the apparent offset between the theoretical and experimental curves is that the $E$ band sampling method does not fully separate out the larger, overlapping $D$ band contribution. An alternative, to be examined below, is that the $D^{2} \mathrm{~A}_{\mathrm{u}}-E^{2} \mathrm{~A}_{\mathrm{g}}$ states are vibronically coupled, with consequent deviation from the uncoupled state $\beta$-parameter calculations appearing in Fig. 4.

The $F / G$ state subpanel in Fig. 4 shows that now both experimental and theoretical $\beta$-parameters have the characteristic $\sigma$-bond character, namely a much slower rise in $\beta$ from the low threshold value as the energy increases. The $\left(8 b_{u}\right)^{-1} /\left(8 a_{g}\right)^{-1}$ ordering predicted by the HF/Koopmans' approximation is reversed by those calculations that provide a better treatment of electron correlation (Table 2) but we make no attempt to distinguish experimentally the overlapped $8 b_{u} / 8 a_{g}$ contributions.

Summarising, the $X$ and $F / G$ bands show no indications of a chlorine like Cooper minimum, whereas the other outer valence bands of trans-dichloroethene do. The depth of the Cooper minima observed in the experimental $\beta$-parameters is consistent with that in the calculated $\beta$ s, and clearly reflects the amount of atomic-like lone pair character predicted for each molecular orbital. It has been suggested from structure in the high harmonic (HHG) spectrum generated in trans-dichloroethene ${ }^{65}$ that the HOMO might also display a Cooper minimum. A priori this seems unlikely from the orbital calculations, and is not supported by the $\beta$-parameter results reported here.

Branching ratios (relative intensities of the electronic bands normalized to unity ${ }^{44}$ ) are shown in Figure 5 where they are compared with theoretical predictions derived from the calculated photoionization cross sections. The experimental $A, B, C$ state ratios show evidence of the $\mathrm{Cl} 3 \mathrm{p}$ Cooper minimum around $40 \mathrm{eV}$, as already inferred from examining the $\beta$-parameters. The summed $F / G$ ratio shows a strong rise around this region. It should be noted, however, that as the ratios are normalised to unity, the dips due to the Cooper minima in the $A, B$ and $C$ state ratios will cause a corresponding rise in the ratios of the other bands. As noted above for the $\beta$-parameters, the lack of separate distinct maxima in the heavily overlapping experimental $D / E$ bands means any attempted separation of these states is very arbitrary. Hence, we compare only the combined $D / E$ experimental branching ratio with the summed $D+E$ calculated branching ratios in Fig. 5 . While, experimentally, the merged $D / E$ ratio is essentially flat across the photon energy range, the calculation does suggest there should be a weak Cooper minimum dip (corroborating the $\beta$-parameter results). Overall, however, the calculated cross sections provide very reasonable agreement for the branching ratios of bands that are strongly affected by Cooper minima, but somewhat overestimate the $X$ and $F / G$ branching ratios in the 25-45 $\mathrm{eV}$ region, perhaps as a coupled consequence of the underestimated $D / E$ ratio in this region.

\section{Vibrationally resolved results}

\section{X band}

Figure 6 displays an expanded view of the $X$ state photoelectron band, with its distinct vibrational structuring. This structure has been readily assigned, with guidance from the PFI vibrational 
results, ${ }^{28,29}$ to three progressions, involving various combinations of the totally symmetric $v_{2}{ }^{+}, v_{3}{ }^{+}, v_{4}^{+}$ and $v_{5}{ }^{+}$modes. These progressions are labelled in Fig. S1 (Supplementary Material) and transition energies are given in Table S4. A 300K Franck-Condon simulation using our calculated MP2/cc-pVTZ harmonic frequencies (Table S3, Supplementary Material) corroborates this assignment and is included for comparison in Fig. 6. The two peaks observed below $9.6 \mathrm{eV}$ are clearly identified as hot band excitations, predominantly from the thermally excited $v_{5}$ mode of the neutral, and disappear in a $0 \mathrm{~K}$ simulation. A further expanded examination of the $300 \mathrm{~K}$ simulation, in the near threshold region below $9.65 \mathrm{eV}$ (Figure S2 Supplementary Material), indicates that, unlike in the cis- isomer, ${ }^{3}$ the $^{2}$ nominal adiabatic ionization peak is not significantly skewed by underlying transitions from thermally excited levels. Hence, the position of the experimental peak is anticipated to coincide with the true adiabatic transition to within $0.5 \mathrm{meV}$. While the relative peak intensities of the $300 \mathrm{~K}$ FC simulation are not in exact agreement with those in the experimental spectrum, the overall match with experiment is nevertheless very convincing.

Our $X$ state photoelectron band yields vibrational energies of 179,116 and $45 \mathrm{meV}$ for the $v_{2}^{+}, v_{4}{ }^{+}$and $V_{5}{ }^{+}$modes, respectively, based upon the spacing between the peak due to the adiabatic transition and that associated with the first member in each progression. The corresponding energies reported by Woo et $\mathrm{al}^{28}$ are 180.0, 117.0 and $45.5 \mathrm{meV}$, respectively. Our vibrational simulation predicts that the peak observed at $9.793 \mathrm{eV}$ (Table S4) contains a contribution from the $4{ }^{1} 5^{1}$ transition in addition to that from the $3^{1}$ transition. Thus, the spacing between this peak and that due to the adiabatic transition is slightly greater than the vibrational energy $(156.0 \mathrm{meV})$ obtained for the $\mathrm{V}_{3}{ }^{+}$mode in the PFI studies. ${ }^{28}$

The experimental photoelectron anisotropy parameter is also shown in Fig. 6 to illustrate the variation in the $\beta$-parameter, as a function of binding energy, across the $X$ state band. The $\beta$-parameter is seen to have a virtually constant value at this photon energy, and indeed at all other photon energies examined, independent of vibrational level. This $\beta$-parameter behaviour is then also as expected within the Frank-Condon approximation. Overall, the $x{ }^{2} A_{u}$ band ionization appears to be fully consistent with the Born-Oppenheimer treatment embodied in the Franck-Condon approximation.

\section{2. $A / B$ bands}

The $A / B$ band region is examined more closely in Figure 7. The excited ion state harmonic frequencies required for a vibrational simulation were calculated using either the TD-B3LYP or the EOM-CCSD methods and the optimized geometries and frequencies appear in Supplementary Material as Tables S5 and S6 respectively. The TD-B3LYP vibrational calculations for the $A{ }^{2} A_{g}$ state in $C_{2 h}$ symmetry returned an imaginary frequency of $b_{\mathrm{u}}$ symmetry (corresponding to the $\mathrm{C}-\mathrm{Cl}$ antisymmetric stretch), indicating that the true potential minimum was not found. While this might instead suggest a propensity of the ${ }^{2} A_{g}$ state cation for distortion along this normal mode coordinate we failed to locate a lower-lying global true minimum even when symmetry constraints in the calculation are relaxed to permit the lower $\mathrm{C}_{s}$ geometry. However, the $\mathrm{C}_{2 \mathrm{~h}}$ EOM-CCSD calculation did not display these problems, and so it is possible that the apparent symmetry breaking in the TD-B3LYP calculations may be an artefact. $^{66}$

An $A$ band FC simulation obtained from the EOM-CCSD harmonic analysis is included in Figure 7. Superficially, this plot gives a favourable impression of the simulation; while the origin peak is relatively too intense, the next four peaks are reproduced, and with approximately the correct relative intensity. However, there is a major caveat concerning the frequency scaling adopted for the 
simulation in Fig. 7. Although it is standard practice to scale calculated harmonic frequencies to compensate for recognised deficiencies in both the harmonic approximation itself and the adopted method/basis, tabulations of the recommended factors derived by systematic studies are almost all $>0.9$ and most typically fall in the range $0.95-1.0 .{ }^{67-69}$ We are not aware of any systematic study covering the EOM-CCSD/cc-pVTZ frequency calculations, but the scaling factor of 0.83 , empirically identified by matching to the experimental spectrum in Fig. 7, appears then quite extraordinary and unexpected.

The TDDFT and EOM-CCSD $B$ band calculations do, however, produce very similar results for frequencies of the key fully symmetric modes $v_{2}{ }^{+}-v_{5}{ }^{+}$(Supplementary Material Table S7) and this further translates to yield very similar FC simulations. Hence, any justifiable corrective scaling factors must be effectively identical for both. Both simulations are plotted in Fig. 7 with a scaling of 0.97 as recommended for B3LYP/6-311G(d,p) calculations. ${ }^{68,69}$ (This, incidentally, further casts suspicion on the $A$ band EOM-CCSD simulation scaling.) In practice, the choice of precise scaling factor for the $B^{2} B_{g}$ state is a moot consideration as the experimental $B$ band region does not at all resemble its simulations.

The experimental $\beta$-parameters, also plotted in Fig. 7, display significant variations across the $A / B$ region bands. There is a marked increase in the $\beta$ value at a binding energy of $12.0 \mathrm{eV}$, which we have assumed is the onset of the $B$ state ionization. However, below this energy, in the $A$ state region, $\beta$ makes an even bigger step-function increase between the apparent origin peak (labelled $o$ in Fig. 7) at $11.842 \mathrm{eV}$, and the second major peak (labelled $p$ ) at $11.919 \mathrm{eV}$. The lower anisotropy displayed by peak $o$ is a behaviour that is maintained through the Cooper minimum, and across the full photon energy range (Figure 8(a)). This figure also shows that the $\beta$-parameters associated with the other peaks $(p-t)$ exhibit a pronounced dip due to the Cooper minimum. The corresponding vibrational branching ratios, plotted in Fig. 8(b), likewise show that in the Cooper minimum region the behaviour displayed by peak $o$, the nominal A state origin, is quite idiosyncratic.

As first suggested by Domcke, ${ }^{2}$ vibronically induced intensity borrowing can also signify that the differential cross section (angular distribution) acquires characteristics associated with the other, interacting state. As a result, the $\beta$-parameter can vary with the extent of vibronic interaction occurring across a photoelectron band, thereby contradicting the FC assumption that $\beta$ should remain invariant. Consequently, our $\beta$-parameter observations may be interpreted as revealing an onset of strong vibronic mixing falling between peaks $o$ and $p$.

Summarising, we conclude that experimental results for the merged $A / B$ band in trans-dichloroethene do not accord with simple Franck-Condon model expectations. The FC simulations of vibrational structure (relative intensities, peak spacings) are not wholly convincing, certainly not in the $B$ state region, nor does the vibrationally resolved photoelectron anisotropy show the simple decoupled, invariant behaviour predicted by the FC assumptions. We defer further discussion of the evident failure of the simple Franck-Condon modelling until Section III.D.3

\section{C band}

The $C$ state photoelectron band, plotted in Figure 9, superficially presents a rather regular vibrational structure, and empirically we can identify progressions in a $740 \mathrm{~cm}^{-1}$ vibrational mode, built upon 0 , 1 , or (more tentatively) 2 quanta excitation of a $280 \mathrm{~cm}^{-1}$ mode. By comparison with calculated frequencies in $\mathrm{C}_{2 \mathrm{~h}}$ symmetry (Supplementary Material Table S8) these are likely to be, respectively, 
the fully symmetric $v_{4}{ }^{+}\left(\mathrm{C}-\mathrm{Cl}\right.$ stretching) and $\mathrm{v}_{5}{ }^{+}(\mathrm{C}=\mathrm{C}-\mathrm{Cl})$ bending modes. Once again, distinct hot bands are observed below the adiabatic peak at $12.583 \mathrm{eV}$ in the experimental spectrum (Fig. 9).

The TD-B3LYP and EOM-CCSD vibrational calculations for the $\mathrm{C}_{2 \mathrm{~h}} C^{2} \mathrm{~B}_{\mathrm{g}}$ state are reported in Tables S5 and S8. Exactly as we found for the $A\left(10 \mathrm{a}_{\mathrm{g}}\right)^{-1}$ state calculations, constraining the geometry to the same $\mathrm{C}_{2 \mathrm{~h}}$ symmetry as the neutral molecule in the TD-B3LYP calculation produces one imaginary frequency of $b_{\mathrm{u}}$ symmetry, suggesting that a distorted planar $\mathrm{C}_{\mathrm{s}}$ geometry would be more stable. However, it was again not possible to locate a lower, global minimum even with relaxed symmetry constraints, and this symmetry breaking is likewise probably a computational artefact. ${ }^{66}$ Again, also, the EOM-CCSD calculations were not so affected, and the 300K FC simulation using these harmonic normal modes is shown in Fig. 9.

The FC simulation very well corroborates the empirical assignment of the strongest peaks as a progression comprised principally of the $v_{4} a_{\mathrm{g}}$ mode, both in terms of spacing and relative intensities. This does not extend to the intermediate experimental peaks, empirically assigned to combinations with one or two quanta excitation in the $v_{5} a_{\mathrm{g}}$ mode. Neither the position, and certainly not the intensity, of these peaks is adequately reproduced by the FC simulation. We anticipate that this may flag some vibronic interaction and defer further consideration until Section III.D.

The $\beta$-parameter (Fig. 9) displays fairly constant behaviour across the $\mathrm{C}$ band, except in the region of the hot bands, below $12.56 \mathrm{eV}$, where it drops very significantly. The shallow decrease in $\beta$ across the rest of the band structure can be attributed to a reduction of electron kinetic energy as the vibrational excitation increases; as may be observed in the non-vibrationally resolved $\beta$-parameter (Fig. 4), around $h v=22 \mathrm{eV} \beta(\mathrm{E})$ has positive gradient. Consequently, and with no need to invoke vibronic interactions, as the $C$ state's vibrational(electron kinetic) energy increases(decreases) across the photoelectron band the $\beta$-parameter measured with a photon energy of $22 \mathrm{eV}$ can be expected to decrease(increase) because of this $\beta(E)$ gradient. Conversely, at $h v=40 \mathrm{eV}$ where the fixed equilibrium geometry $\beta(E)$ has the opposite (negative) gradient entering into the CM dip (Fig. 4), a very slight increase in the value of $\beta$ with increasing vibrational excitation resolved across the majority of the $C$ band is observable. Unlike the vibrational structure, which is irregular, the $C^{2} \mathrm{~B}_{\mathrm{g}} \beta$-parameters do not immediately appear to display significant deviations from Franck-Condon assumptions.

4. D/E Bands

The $D / E$ state photoelectron band, shown in Figure 10, displays extended vibrational structure superimposed on a broad continuum, with the $E$ state appearing to contribute as a shoulder to high binding energy. Although regular progressions cannot be identified, the structure exhibits numerous short series with spacings of $\sim 35-45 \mathrm{meV}$. The EOM-CCSD and TD-B3LYP vibrational calculations (Supplementary Material Tables S9 and S10) show the excited $D\left(2 \mathrm{a}_{u}\right)^{-1}$ state adopting a twisted geometry $\left(C_{2}\right.$ symmetry). Loss of planarity stabilises the $D$ state ion by $7 \mathrm{~kJ} \mathrm{~mol}^{-1}$ (EOM-CCSD/pVTZ) to $10 \mathrm{~kJ} \mathrm{~mol}^{-1}$ (TD-B3LYP/6-311G(d,p)) and is readily rationalised as the ionization removes an electron from the $\mathrm{C}=\mathrm{C} \pi$ orbital.

The vibrational simulations (Fig 10) for the $\left(2 \mathrm{a}_{\mathrm{u}}\right)^{-1}$ ionization achieve a good qualitative agreement with experiment, capturing the rather unique vibrational character of this band when compared and contrasted with those found at lower binding energies. Because of the big planar-twisted geometry change on ionization, one can expect a significant range of vibrational excitations. Prominent among these, as indicated by the $\mathrm{FC}$ simulations, are the $\mathrm{v}_{4}{ }^{+} a$ (H-atom wag) and $\mathrm{v}_{5}{ }^{+} a(\mathrm{Cl}-\mathrm{C}-\mathrm{C}-\mathrm{Cl}$ def.) modes, 
but with extensive combination bands producing the more irregular appearance. Consideration of possible contribution made by the $\left(9 \mathrm{ag}_{\mathrm{g}}\right)^{-1}$ ionization is deferred to Section III.D.3.

There is a strong variation of $\beta$ across the $D / E$ band(s), with the value near the high energy end of the band being approximately half that in the peak. There are strong indications that a quantitative treatment of the photoionization dynamics is not available within the simple Franck-Condon model, and that a coupled $D-E$ state simulation is required.

\section{5. $F / G$ Bands}

For completeness, we show in Figure 11 an expanded view of the $F / G$ bands, and the associated $\beta$ parameters. The vibrational structure on the low energy side of the band appears to consist of two short progressions, each involving excitation of a mode having an energy of $\sim 95 \mathrm{meV}\left(766 \mathrm{~cm}^{-1}\right)$, with one of the progressions having an additional excitation of another mode with an energy of $\sim 40$ meV $\left(323 \mathrm{~cm}^{-1}\right)$. The alternating peak intensity in this region is reflected by the well resolved alternations in the $\beta$-parameter. Towards higher binding energy, the vibrational structure becomes increasingly complex and the value of the corresponding $\beta$-parameter steadily decreases. The inset to Fig. 11 shows results recorded at the higher photon energy of $46 \mathrm{eV}$. The mean $\beta$ value changes with photon energy, as already established, but it is further evident that the variation in $\beta$ across the band envelope is also now different. Given this evidence as a clear indication of the Franck-Condon breakdown, and the possibility that an independent electron model of ionization is also breaking down for these deeper lying orbitals, we make no attempt at a vibrational simulation for now.

\section{Vibronic Interaction}

\section{Tuning and coupling modes}

In Tables 4 and 5 we present vibronic coupling constants $\kappa_{i}^{s}$ and $\lambda_{i j}^{s}$ for the five lowest excited states of the trans-dichloroethene radical cation. Inspection of the $\kappa_{i}^{s}$ and $\lambda_{i j}^{s}$ values allows one to understand which modes are most important for the vibronic coupling problem under study. The totally symmetric modes have to be considered since they tune the intersection of the potential energy surfaces, and the non-totally symmetric modes are responsible for the coupling of the surfaces. ${ }^{1}$ In these tables we present also the Poisson parameters $a$, which are more informative than the coupling constants, since they directly characterize the maximal extent to which the modes are excited.

It follows from the magnitudes of the calculated $a$ values (Table 4), that the totally-symmetric modes $v_{2}{ }^{+}-v_{5}{ }^{+}$should be taken into account for proper treatment of vibronic coupling of the $A^{2} A_{g}, B{ }^{2} B_{u}$, and $C^{2} \mathrm{~B}_{\mathrm{g}}$ states as at least one of them is active in each of the potentially coupled states. The $\mathrm{C}-\mathrm{Cl}$ stretching mode $V_{4}{ }^{+}$is seen to be particularly important for the $A^{2} A_{g}$ and $C^{2} B_{g}$ states, as empirically corroborated by the long $v_{4}^{+}$progression observed in the $C$ state spectrum (Fig 9). Likewise, the first vibrationally excited peak ( $11.92 \mathrm{eV}$ ) in the $A$ band region (Fig. 7 ) is attributed by the EOM-CCSD FC simulation to the $v_{4}{ }^{+}$mode. For the $B{ }^{2} \mathrm{~B}_{\mathrm{u}}$ state, Table 4 indicates that the $\mathrm{CCCl}$ angle deformation mode $v_{5}{ }^{+}$is be particularly important. All five totally-symmetric modes are expected to be active in the $D^{2} \mathrm{~A}_{\mathrm{u}}$ and $E{ }^{2} \mathrm{~A}_{\mathrm{g}}$ states, including (somewhat atypically) the $\mathrm{C}-\mathrm{H}$ stretching mode $\mathrm{v}_{1}{ }^{+}$. The most active modes here are again the low-frequency modes $v_{4}{ }^{+}$and $v_{5}{ }^{+}$. This picture differs from the one 
observed in cis-dichloroethene ${ }^{4}$ where the $v_{4}{ }^{+}$and $v_{5}{ }^{+}$modes dominated in all states while the remaining totally-symmetric modes were comparatively less important.

As seen from Table 5 , certain interstate coupling constants, $\lambda$, could not be determined. The reason for this is that the present LVC model could not be fitted to the potential energy surfaces of the interacting state along the coordinates of the respective modes. A similar situation was encountered in our studies of cis-dichloroethene ${ }^{4}$ but in that case we could, however, estimate approximate $\lambda$ constants from the single (lowest) surface. In the present study of the trans-isomer this approach unfortunately turned out to be inapplicable. Thus, the present dynamics calculations were performed whilst exempting modes for which we failed to identify $\lambda$ constants, i.e., the $v_{12}{ }^{+}\left(b_{\mathrm{u}}\right)$ and $v_{7}{ }^{+}\left(a_{\mathrm{u}}\right)$ modes for the $A-B-C$ states, and the $v_{7}^{+}\left(a_{\mathrm{u}}\right)$ mode for the $D-E$ states. The formally calculated negative Poisson parameters for these interactions (Table 5 ) are unphysical, but their magnitude can still be informative to characterise the energy changes along the respective coordinates. As seen from the values obtained, the effects of the neglected modes can in principle be appreciable. However, these effects can be recovered only in higher-order models, beyond the present LVC approximation.

Disregarding the excluded modes, the general coupling situation in trans-dichloroethene is quite similar to that in cis-dichloroethene. ${ }^{4}$ The lowest two excited cationic states, $A{ }^{2} A_{g}$ and $B{ }^{2} B_{u}$, are moderately coupled by the two modes, $\mathrm{v}_{10}{ }^{+}\left(b_{\mathrm{u}}\right)$ and $\mathrm{v}_{11}{ }^{+}\left(b_{\mathrm{u}}\right)$, describing in-plane $\mathrm{C}-\mathrm{H}$ bending and $\mathrm{C}$ $\mathrm{Cl}$ stretching deformations, respectively. The role of the low-frequency $\mathrm{CCCl}$ angle deformation mode, $v_{12}{ }^{+}\left(b_{u}\right)$, remains, as discussed above, so far unclear. The second and the third lowest excited states, $B^{2} \mathrm{~B}_{\mathrm{u}}$ and $C^{2} \mathrm{~B}_{\mathrm{g}}$, are appreciably coupled via the $\mathrm{v}_{6}^{+}\left(a_{\mathrm{u}}\right)$ out-of-plane $\mathrm{CH}$ bending mode, whereas the role of the low-frequency out-of-plane torsion mode $v_{7}^{+}\left(a_{\mathrm{u}}\right)$ mode in their coupling is again unclear. Finally, there is a definite $A^{2} \mathrm{~A}_{\mathrm{g}}-C^{2} \mathrm{~B}_{\mathrm{g}}$ coupling via the out-of-plane $\mathrm{CH}$ bending mode $v_{8}^{+}\left(b_{\mathrm{g}}\right)$.

Just as in cis-dichloroethene, the $D$ and $E$ states in trans-dichloroethene are strongly coupled. However, in contrast to the cis-isomer, this coupling in the trans-isomer is firmly established only via one mode, $v_{6}{ }^{+}\left(a_{\mathrm{u}}\right)$, while the role of the second coupling mode, $v_{7}^{+}\left(a_{\mathrm{u}}\right)$, has yet to be understood (Table 5).

\section{Potential energy surfaces}

Another important aspect of vibronic coupling concerns the potential energy surfaces of the interacting states. More specifically, the energy gaps separating the electronic states and the minimal energies of their conical intersections ( $\mathrm{Cls}$ ) play a decisive role in defining the spectral domains where the adiabatic approximation breaks down (Table 6).

The $A, B$, and $C$ states are compactly grouped $(\sim 0.8 \mathrm{eV})$, just as in cis-dichloroethene. ${ }^{4}$ The vertical energy intervals within the group are however somewhat modified: the $A-B$ interval is slightly decreased and the $B-C$ interval is slightly increased $(\sim 0.1 \mathrm{eV})$. The vertical gap between the $D$ and $E$ interacting states is slightly reduced compared to that in the cis-isomer and amounts to only $0.17 \mathrm{eV}$. Such small vertical separations of the states within the $(A-B-C)$ and the $(D-E)$ groups should necessarily lead to a certain type of adiabatic approximation breakdown.

Before discussing further characteristics related to the potential energy surfaces, we recall that whereas the present vertical ionization energies are obtained from our OVGF $a b$ initio calculations, the adiabatic transition energies and the minimal energies of conical intersections are estimated from the present LVC model using analytical expressions presented in Ref. 1 (Eqs. (3.23)-(3.26), (3.31) on pages 130-133). 
As can be seen (Table 6 ), the $A^{2} A_{g}$ state of trans-dichloroethene is involved in the conical intersection with the $B{ }^{2} \mathrm{~B}_{\mathrm{u}}$ state, with a minimal intersection energy of $12.07 \mathrm{eV}$. Since the $\mathrm{Cl}$ is located $\sim 0.2 \mathrm{eV}$ above the adiabatic minimum of the $A^{2} A_{g}$ state potential energy surface (characterized by a transition energy of $11.83 \mathrm{eV}$ ) and the non-adiabatic effects become important only near and above the $\mathrm{Cl}$, one can expect that for a certain part of the $A-B-C$ spectrum, at low energy, the ordinary Franck-Condon approximation should be valid. On the other hand, the $\mathrm{Cl}$ is located in energy almost directly at the adiabatic minimum of $B{ }^{2} B_{u}$ state $(12.01 \mathrm{eV})$, so that the entire spectrum of the $B^{2} B_{u}$ state should lie within the domain of non-adiabatic dynamics. These inferences concur with the conclusions drawn from the FC vibrational structure simulations shown in Fig. 7, despite the caveat about the required extreme scaling of the $A$ state harmonic frequencies. The step change in the $\beta$ anisotropy parameter observed at $\sim 11.88 \mathrm{eV}$ (Fig. 7) might, however, be taken to indicate a lower onset for vibronic interaction in the $A-B$ band region.

The $C^{2} \mathrm{~B}_{\mathrm{g}}$ state is involved in Cls with both the $A^{2} \mathrm{~A}_{\mathrm{g}}$ and $B^{2} \mathrm{~B}_{\mathrm{u}}$ states. The minimal energy of the $C^{2} \mathrm{~B}_{\mathrm{g}}$ $A^{2} \mathrm{~A}_{\mathrm{g}} \mathrm{Cl}$ is $13.12 \mathrm{eV}$, which is well above the adiabatic minimum of the $C^{2} \mathrm{~B}_{\mathrm{g}}$ state at $12.61 \mathrm{eV}$. The $C^{2} \mathrm{~B}_{\mathrm{g}}-B^{2} \mathrm{~B}_{\mathrm{u}}$ intersection takes place at an even higher energy of $13.70 \mathrm{eV}$. In view of the moderate coupling strength mentioned above, the spectral envelope of the $C^{2} B_{g}$ state might therefore be relatively unaffected by non-adiabatic effects, again concurring with the partially successful FranckCondon simulation for this state (Fig. 9)

The vertical energy gap between the $D^{2} \mathrm{~A}_{u}$ and $E{ }^{2} \mathrm{~A}_{\mathrm{g}}$ states is $0.17 \mathrm{eV}$. The states become nearly degenerate when their $E_{0-0}$ transition energies are considered (13.69 and $13.68 \mathrm{eV}$, at the $C_{2 h}$ molecular configuration). Our LVC model predicts that the $D^{2} A_{u}$ state should develop a potential energy surface with a double-minima along the coordinate of the $v_{6}\left(a_{u}\right)$ mode, as a result of the strong vibronic coupling with the $E^{2} \mathrm{Ag}$ state, characterized by a stabilization energy of $0.017 \mathrm{eV}$. A similar symmetry-breaking was predicted by our calculations for the analogous pair of states in cisdichloroethene. ${ }^{4}$ In trans-dichloroethene this yields the low-symmetry $C_{2}$ point group structure as the true minimum of the potential energy surface, whereas the symmetric $D_{2 h}$ structure appears as a saddle point connecting the $\mathrm{C}_{2}$ minima. This picture agrees very well with the results of the direct geometry optimization calculations discussed above.

The $D^{2} \mathrm{~A}_{\mathrm{u}}-E^{2} \mathrm{~A}_{\mathrm{g}}$ minimal $\mathrm{Cl}$ energy predicted by the LVC model is $13.8 \mathrm{eV}$, which is slightly above $(\sim 0.1 \mathrm{eV})$ the adiabatic minima of both states. Most of the overlapping photoelectron band falls above the intersection point and so nuclear dynamics will occur on both interacting surfaces in a non-adiabatic mode. This implies that the effects of vibronic coupling will be particularly strong in the spectral band system formed by these two states.

\section{Simulation of Vibronic Spectra}

The numerical results from our LVC model (Tables $4-6$ ) have successfully corroborated the possibilities for vibronic interaction that were inferred experimentally in Section III.C, and clearly identify the mechanisms that would permit this. One form of evidence driving the experimental inferences was an observed deviation of the vibrational structure from Franck-Condon expectations. It is then natural to examine how simulations using the LVC model may overcome the FC limitations. We first recall that, as explained in section II.B, the current LVC model describes vibronic transition energies with only ground state vibrational parameters. The final-state totally symmetric vibrational modes are effectively approximated with ground state frequencies - unlike the FC simulations (Section III.C) where the excited state vibrational parameters are explicitly calculated. Hence, we only 
expect qualitative agreement with experiment from the first order LVC model. Nevertheless, insight into the influence of vibronic coupling can be gained by comparing the so-called Poisson spectrum, which excludes the interstate coupling terms, with the "full" vibronic simulation. Comparison of both with experiment also helps to better appreciate the manifestations of vibronic coupling in each studied photoelectron band.

\section{The $A{ }^{2} A_{g} / B^{2} B_{u}$ and $C^{2} B_{g}$ spectra}

The results of our vibronic modelling of the $\left(A^{2} A_{\mathrm{g}}-B^{2} \mathrm{~B}_{\mathrm{u}}-C^{2} \mathrm{~B}_{\mathrm{g}}\right)$ state band system are shown in Figure 12 where the theoretical spectrum accounting for vibronic coupling (Figure $12(b)$ ) is compared with the experimental spectrum (Figure 12(a)), and with the Poisson spectrum(Figure 12(c)). The spectra were aligned so that the position of the peak at lowest binding energy in each spectrum matches. An overall shift of 0.025 and $0.019 \mathrm{eV}$ was applied to the vibronic and Poisson spectra, respectively, which accounts for the mean error of the respective theoretical treatments.

As expected, the Poisson spectrum obtained without the treatment of vibronic coupling does not reproduce several important features of the band observed in the experimental spectrum between binding energies of 11.8 and $12.3 \mathrm{eV}$ - most notably the second most intense peak at $11.9 \mathrm{eV}$ is "missing". By way of contrast, the full vibronic simulation shown in panel (b)) introduces an additional peak at this binding energy, identified as a transition to a vibronic state of $B_{u}$ symmetry. Importantly, this also helps rationalise the large step rise in the $\beta$ anisotropy parameter observed experimentally at this binding energy (Fig.7) and which we previously inferred in section III.C.2 as a possible consequence of vibronic mixing. ${ }^{2}$ While this feature lies below the predicted $12.07 \mathrm{eV}$ energy of the $A-B$ conical intersection (Table 6 ) it reveals a significant role for nonadiabatic dynamics in the general vicinity of such an interaction, often considered as zones where nuclear motion effectively may proceed simultaneously on both intersecting surfaces.

More generally, the full LVC simulation generates much more vibronic structure across the $A / B$ region and achieves a good semi-quantitative agreement with experiment. This especially constitutes a big improvement for binding energies above $12 \mathrm{eV}$, the " $B$ " band, for which the FC simulation in Fig. 7 (and Poisson spectrum in Fig. 12c) fail badly. Although not quantitatively accurate (owing to deficiencies discussed in the preceding paragraphs), the 1st order LVC calculations without doubt reflect the underlying $A / B$ vibronic coupling. Unfortunately, our calculation provides no means for interpreting the final vibronic states (since this would require an explicit evaluation of the corresponding wavefunctions and their transformation from the diabatic to adiabatic basis, ${ }^{1}$ which is not feasible in our case for technical reasons). Thus, at present, the vibronic features cannot be unambiguously assigned.

Moving on to consider the better separated $C$ state, a comparison of the Poisson and vibronic simulated spectra (Figures 12 (c) and 12 (b), respectively) shows the envelope of the $C^{2} B_{g}$ progression is little changed by the vibronic coupling, and indeed both are similar to the FC simulation in Fig. 9. There, in section III.C.3, we were able to assign the principal features to a progression in the fully symmetric $\mathrm{V}_{4}^{+}$mode that is easily reproduced by all the current simulations. However, the weaker intermediate lines, tentatively assigned to excitations of the $v_{5}{ }^{+}$mode, appear shifted, and relatively more intense in the experimental spectrum than in the FC, Poisson, or full LVC simulations. Moreover, in the vibronic simulation, dense clusters of lines built around the allowed Franck-Condon excitations 
of the $a_{g}$ modes, are just discernible. These lines are of $\mathrm{B}_{\mathrm{g}}$ symmetry (shown by blue colour) and therefore belong either to the ${ }^{2} \mathrm{~B}_{u} \times a_{u}$ or ${ }^{2} \mathrm{~A}_{g} \times b_{g}$ vibronic states. As a consequence of their presence, some slight broadening of the lines in the vibronic spectrum can be seen, caused by the interaction with the two lower-lying states.

Our results confirm that the $C^{2} \mathrm{~B}_{\mathrm{g}}$ state is vibronically coupled to the $A^{2} \mathrm{~A}_{\mathrm{g}}$ and $B{ }^{2} \mathrm{~B}_{\mathrm{u}}$ states, but since the $\mathrm{Cl}$ of the respective potential energy surfaces occur well above the adiabatic minimum of the $C^{2} \mathrm{~B}_{\mathrm{g}}$ state, the interactions are, as expected, weak. The $1^{\text {st }}$ order LVC model we have employed suggests that incorporating vibronic effects does not offer an immediate improvement on the moderately successful intensity distribution provided by FC simulation, but this has to be a qualified judgement. It has already been noted that, at the level employed here, reliable vibrational mode parameters not available to treat the excited state dynamics. Additionally, the vibronic modelling of the interaction with the nearest $B{ }^{2} \mathrm{~B}_{u}$ state is necessarily incomplete due to the missing (undetermined) $v_{7}$ interstate coupling constant, $\lambda$ (Table 5).

Vibronic structure of the $D^{2} A_{u} / E^{2} A_{g}$ bands

As in cis-dichloroethene, the $D$ and $E$ states form a single photoelectron band with very complex vibronic structure, located in the 13.65-14.7 eV energy interval (Figure 13(a)). As discussed above, the $D{ }^{2} \mathrm{~A}_{u}$ and $E{ }^{2} \mathrm{~A}_{\mathrm{g}}$ states fully overlap and have virtually identical adiabatic origins. Even at the noninteracting level, this situation gives rise to a highly complex envelope, as apparent from the Poisson spectrum (Figure 13(c)). The overall envelope of the Poisson spectrum matches quite well the width of the experimental $D / E$ band spectrum. However, the experimental curve shows minimally resolved vibronic structure, resembling more the $D^{2} A_{u}$ state FC simulation made with a twisted $C_{2}$ geometry (Fig. 10) in the lower half of the binding energy range, whereas the Poisson model predicts a very structured envelope extending across the full $D / E$ band range.

Enabling the vibronic interaction further complicates the spectrum predicted by the LVC model (Figure $13(\mathrm{~b}))$, especially above the predicted $\mathrm{Cl}$ at $13.8 \mathrm{eV}$. The agreement between the experimental and theoretical spectra improves, especially at higher energy, but there remain certain qualitative distinctions in intensity and resolution at binding energies below the experimental $D / E$ band maximum. The less structured pattern of the experimental envelope in this region can only be explained by the presence of a sufficiently large number of vibronic satellites which are associated here with the excitations of $a_{u}$ coupling modes. The absence of such satellites in our calculations can be related to the limitations of our LVC model, which presumably underestimates the coupling of the $D^{2} A_{u}$ and $E{ }^{2} A_{g}$ states (since, as discussed above, it treats only one of the two available $a_{u}$ coupling modes).

Finally, it should be noted that the present $D^{2} \mathrm{~A}_{\mathrm{u}}-E^{2} \mathrm{~A}_{\mathrm{g}}$ vibronic model might also not be fully complete, since it does not include the ${ }^{2} \mathrm{~B}_{\mathrm{g}}\left(3 \mathrm{a}_{\mathrm{u}}{ }^{-2}(\pi) \mathrm{b}_{\mathrm{g}}\left(\pi^{*}\right)\right)$ shake-up satellite state, predicted by our $\mathrm{ADC}(3)$ calculations (Table 2 ) in the vicinity of the $D{ }^{2} A_{u}$ and $E{ }^{2} A_{g}$ states. The respective vertical ionization energies of the three states are $14.45,13.93$ and $14.15 \mathrm{eV}$, so that they all lie close enough to be vibronically coupled. In Figure 1 it can be seen that the ${ }^{2} \mathrm{~B}_{\mathrm{g}}\left(3 \mathrm{a}_{\mathrm{u}}{ }^{-2} \mathrm{~b}_{\mathrm{g}}\right)$ satellite state contributes to the $D$ $E$ band, though with a rather small photoelectron intensity. In the case of sufficiently strong coupling, the intensity however could be borrowed from the other states, so that a quite different overall spectral envelope of the $D-E$ band could be obtained. 
In this study we have recorded VUV photoelectron spectra and photoelectron angular distributions of trans-dichloroethene over a wide photon energy range. We have been able to identify and assign the photoelectron bands with the aid of calculations, corroborated by the observed photon energy dependence of the photoelectron anisotropy parameter $\beta$ for these bands. This helps distinguish the photoionization of $\sigma$ - and $\pi$ - type orbitals. More particularly, it helps identify the degree of atomiclike character of chlorine lone pair orbitals from the depth of their Cooper minima, observed around $40 \mathrm{eV}$, the energy of the $3 p$ Cooper minimum in atomic chlorine.

All bands below a binding energy of $18 \mathrm{eV}$ have at least some resolved vibrational structure. The first $X\left(3 \mathrm{a}_{u}\right)^{-1}$ state (ionization of the $\mathrm{C}=\mathrm{C} \pi$ orbital) exemplifies the behaviour associated with a fully adiabatic cation. Its vibrational structure is extensive, but regular, and is readily assigned by empirical observation. The intensity distribution is also well reproduced by simulations using calculated harmonic normal modes and the Franck-Condon approximation. Moreover, the photoelectron $\beta$ parameter displays no vibrational level dependence, indicating a full uncoupling of electronic and nuclear motion in this state.

The $X^{2} A_{u}$ state thus sets a benchmark used for preliminary assessment of the other bands' behaviour. With this in mind, in the overlapped $A{ }^{2} A_{g} / B{ }^{2} B_{u}$ region FC simulations reproduce the vibrational structure of the $A$ state rather well, but fail badly across the higher energy $B$ state region. Significantly, however, a step-function rise of the $\beta$ anisotropy parameter just above the $A$ state origin provides an alternative flagging of suspected vibronic interaction. In contrast, the $C^{2} B_{g}$ band is better separated in the spectrum and has a regular vibrational structure that empirically appears to be straightforwardly assigned (as a progression in $v_{4}{ }^{+}$in combination with 0-2 quanta of the $v_{5}{ }^{+}$mode). Also, the experimental $\beta$-parameter is effectively constant across the main area of the band in accordance with the FC approximations. However, there are serious deficiencies in the intensity distribution and in the identification of the $\mathrm{V}_{5}{ }^{+}$mode excitation evidenced in the Franck-Condon simulation of this band. The $D^{2} A_{u}$ and $E{ }^{2} A_{g}$ bands, which are heavily overlapping, have a closely spaced, irregular vibrational structure on the low binding energy side. The qualitatively different experimental appearance is partially captured by a harmonic Franck-Condon simulation, but the $\beta$-parameter again shows a very significant variation with vibrational energy level. Finally, the $F / G$ bands, again strongly overlapping, have a seemingly regular vibrational structure to low binding energy, but with an oscillating $\beta$ parameter across these vibrational peaks. There are, however, suggestions that the independent single particle model for ionization may be starting to break down in this region, and for now further investigation has been restricted.

These initial assessments stimulated a more detailed investigation of vibronic interaction in the $A-B-C$ and $D-E$ states using the linear vibronic coupling model with coupling parameters obtained from $a b$ initio calculations. The calculated vibrational mode coupling parameters and adiabatic potentials are sufficient to confirm the likelihood of vibronic interaction within these groups of states, and to identify the mechanisms that allow this to occur. For the $A^{2} A_{g}$ and $B{ }^{2} B_{u}$ states a low-lying conical intersection was identified that enables a non-adiabatic coupling between these states, resulting in a single $A / B$ photoelectron band that should be appreciably influenced by the vibronic interaction. This is confirmed by the identification of vibronically induced satellite transitions throughout the $A / B$ band region by the LVC simulations and a much more realistic looking simulation of the $B$ state region. 
However, having claimed a confirmation of the vibronic interaction across the overlapped $A^{2} A_{g} / B^{2} B_{u}$ band it may then seem inconsistent that the $A$ state FC vibrational simulation (Fig. 7) appears to show much better agreement with experiment for the lower binding energy region than does the LVC calculation that incorporates a treatment of the vibronic coupling. First, one must recall the caveat, that the harmonic scaling factor required to bring the FC simulation into such seemingly good agreement is unexpectedly small. Nevertheless, it seems unlikely that such close agreement is purely coincidental. Perhaps the difficulties noted with converging the adiabatic potential (with a tendency to deform along a $b_{\mathrm{u}}$ symmetry mode) and the exaggerated anharmonicity that the scaling might imply, are in some sense diagnostic of the distortion of the adiabatic harmonic potential by the nearby conical intersection. In all events the most significant experimental signature of vibronic interaction is found not in the vibrational structure, but in the step-function increase of the $\beta$-parameter that occurs just above the $A$ state origin peak. This is an expected consequence of vibronic intensity borrowing, ${ }^{2}$ and is strongly confirmed to coincide with the onset of vibronically induced features in the LVC simulation. Within its acknowledged limitations, the LVC simulation unambiguously confirms the dominance of vibronic structure in the $B$ state region lying above the predicted conical intersection.

The $C$ state band is also shown by the LVC coupling constants to be affected by vibronic coupling within this group, although to a lesser degree and hence with less impact on the observed spectrum. A relatively flat experimental $\beta$-parameter curve offers no hint of non-FC behaviour. Nevertheless, an irregular vibrational peak intensity pattern is readily identified in the experiment by comparison with all three simulations (Poisson, LVC, FC). That LVC spectrum simulation fails, however, to provide a significantly improved quantitative agreement with experiment over the non-coupled Poisson and FC models is likely attributable to the missing $v_{7}$ coupling constant and reliance on only ground state frequencies in the current first order LVC calculation.

Stronger vibronic interaction is predicted for the heavily overlapped $D^{2} \mathrm{~A}_{\mathrm{u}}$ and $E^{2} \mathrm{~A}_{\mathrm{g}}$ states by the LVC calculations. The adiabatic minima of these two states are found to be almost degenerate in energy with strong interstate coupling terms. Consequently, the potential energy surface of the $D^{2} \mathrm{~A}_{u}$ state is predicted to have a double-minimum shape with respect to the $a_{\mathrm{u}}$ deformations of the molecular structure and a single photoelectron band is expected to result. Similar circumstances arose in our earlier study of the cis- isomer. ${ }^{3,4}$ Experimentally, both isomers display a long progression in the lower energy region, arising from a twisting distortion in the cation, and which is reasonably well modelled in the FC simulations. The better resolved structure afforded in the cis-isomer spectrum nevertheless reveals a clearly irregular structure around $0.1 \mathrm{eV}$ above the band origin, that is not so readily discerned in the less well defined band of the trans- isomer. The LVC vibrational simulation for the trans- isomer not unexpectedly predicts an irregular vibrational structure that reflects non-adiabatic nuclear dynamics occurring on the two coupled potential energy surfaces, but without achieving a fully quantitative match to experiment. However, for both isomers, a similar variation of the $\beta$ parameter across the $D / E$ band provides an alternative indicator of vibronic interaction, and further helps corroborate the essential coupling identified by the LVC model.

At the level employed here, the LVC simulations are not expected to provide fully realistic simulations of the experimental spectra. The limitations of current restriction to a first order treatment are evident in the difficulties encountered in fitting the potentials and the consequent failure to extract three needed interstate coupling constants. Secondly, higher order potential fitting would be required to accurately reproduce realistic excited state frequencies. While acknowledging these deficiencies in the current model, these LVC calculations successfully confirm the inferences made by considering 
apparent limitations of the Franck-Condon modelled vibrational structure and/or information from vibrationally resolved photoelectron angular distributions. It is also salutary to realise how extensively the spectra and properties of even relatively small molecules like the dichloroethenes are dominated by vibronic interaction.

\section{SUPPLEMENTARY MATERIAL}

See supplementary material for further presentation of experimental spectra, tables for computed geometry and vibrational modes and a table giving measured cation vibrational energies.

\section{ACKNOWLEDGEMENTS}

This research was undertaken as part of the ASPIRE Innovative Training Network, which has received funding from the European Union's Horizon 2020 research and innovation programme under the Marie Sklodowska-Curie Grant Agreement No. 674960. AD acknowledges an ESR fellowship provided by Aspire.

We acknowledge the provision of beamtime by Synchrotron Soleil (beamtime Proposal No. 20171402) and we thank the technical staff at Soleil for their support and for the smooth operation of the facility.

A.B.T., E.K.G. and A.D.S. gratefully acknowledge the grant, Grant No. FZZE-2020-0025, from the Ministry of science and higher education of the Russian Federation. DMPH is grateful to the Science and Technology Facilities Council (United Kingdom) for financial support.

The University of Nottingham High Performance Computing Facility provided computational resources partially supporting this investigation.

Hassan Ganjitabar is thanked for help with the data acquisition.

\section{DATA AVAILABILITY}

Raw data were generated at the Synchrotron Soleil large scale facility. Derived data supporting the findings of this study are available from the corresponding author upon reasonable request. 


\section{REFERENCES}

H. Köppel, W. Domcke, and L. S. Cederbaum, Adv. Chem. Phys. 57, 59 (1984). W. Domcke, Phys. Scr. 19, 11 (1979). I. Powis, R. C. Menzies, D. M. P. Holland, A. B. Trofimov, A. D. Skitnevskaya, E. V. Gromov, E. Antonsson, M. Patanen, C. Nicolas, and C. Miron, J. Chem. Phys. 149, 074305 (2018).

A. B. Trofimov, I. Powis, R. C. Menzies, D. M. P. Holland, E. Antonsson, M. Patanen, C. Nicolas, C. Miron, A. D. Skitnevskaya, E. V. Gromov, and H. Köppel, J. Chem. Phys. 149, 074306 (2018). D. M. P. Holland, S. Nandi, C. Nicolas, J. D. Bozek, M. Patanen, and I. Powis, Chem. Phys. 542, 111050 (2021).

H. Köppel, W. Domcke, and L. S. Cederbaum, in Conical Intersections: Electronic Structure, Dynamics and Spectroscopy, edited by W. Domcke, D. R. Yarkony, and H. Köppel (World Scientific, Singapore, 2004), pp. 323.

G. A. Worth and L. S. Cederbaum, Annu. Rev. Phys. Chem. 55, 127 (2004).

A. B. Trofimov, H. Köppel, and J. Schirmer, J. Chem. Phys. 109, 1025 (1998).

A. B. Trofimov, A. D. Skitnevskaya, E. K. Grigoricheva, E. V. Gromov, and H. Köppel, J. Chem. Phys. 153, 164307 (2020).

A. Ghosh, K. Rajak, A. K. Kanakati, and S. Mahapatra, Comput. Theor. Chem. 1155, 109 (2019).

R. Sarkar, D. Baishya, and S. Mahapatra, Chem. Phys. 515, 679 (2018).

K. Rajak, A. Ghosh, and S. Mahapatra, J. Phys. Chem. A 122, 8612 (2018).

R. Sarkar and S. Mahapatra, J. Chem. Phys. 147, 194305 (2017).

M. A. Parkes, S. Ali, C. R. Howle, R. P. Tuckett, and A. E. R. Malins, Mol. Phys. 105, 907 (2007).

K. Wittel and H. Bock, Chem. Ber.-Recl. 107, 317 (1974).

N. Jonathan, K. Ross, and V. Tomlinson, Int. J. Mass Spectrom. Ion Phys. 4, 51 (1970).

R. F. Lake and H. Thompson, Proc. R. Soc. London, Ser. A 315, 323 (1970).

A. Katrib, T. P. Debies, R. J. Colton, T. H. Lee, and J. W. Rabalais, Chem. Phys. Lett. 22, 196 (1973).

K. Kimura, S. Katsumata, Y. Achiba, T. Yamazaki, and S. Iwata, Handbook of Hel Photoelectron Spectra of Fundamental Organic Compounds. (Japan Scientific Societies Press, Tokyo, 1981).

W. von Niessen, L. Åsbrink, and G. Bieri, J. Electron Spectrosc. Relat. Phenom. 26, 173 (1982). A. Berndtsson, E. Basilier, U. Gelius, J. Hedman, M. Klasson, R. Nilsson, C. Nordling, and S. Svensson, Phys. Scr. 12, 235 (1975).

J. F. Ying and K. T. Leung, J. Electron Spectrosc. Relat. Phenom. 63, 75 (1993).

L. Mei, M. Chuaqui, C. P. Mathers, J. F. Ying, and K. T. Leung, Chem. Phys. 188, 347 (1994).

N. A. Pradie and H. V. Linnert, 111, 4836 (2007).

K. Takeshita, J. Chem. Phys. 110, 6792 (1999).

V. Galasso, Theochem-J. Mol. Struct. 337, 249 (1995).

R. Locht, D. Dehareng, and B. Leyh, J. Quant. Spectrosc. Radiat. Transf. 251, 107048 (2020).

H. K. Woo, P. Wang, K. C. Lau, X. Xing, and C. Y. Ng, J. Phys. Chem. A 108, 9637 (2004).

Y. J. Bae, M. Lee, and M. S. Kim, J. Phys. Chem. A 110, 8535 (2006).

D. M. P. Holland, I. Powis, G. Öhrwall, L. Karlsson, and W. von Niessen, Chem. Phys. 326, 535 (2006).

D. M. P. Holland, L. Karlsson, and W. von Niessen, J. Electron Spectrosc. Relat. Phenom. 113, 221 (2001).

J. W. Cooper, Phys. Rev. 128, 681 (1962).

S. T. Manson, A. Msezane, A. F. Starace, and S. Shahabi, Phys. Rev. A 20, 1005 (1979).

T. A. Carlson, M. O. Krause, W. A. Svensson, P. Gerard, F. A. Grimm, T. A. Whitley, and B. P. Pullen, Z. Phys. D 2, 309 (1986).

R. M. Rao, E. D. Poliakoff, K. H. Wang, and V. McKoy, Phys. Rev. Lett. 76, 2666 (1996).

I. Powis, M. Patanen, E. Antonsson, C. Nicolas, C. Miron, and D. M. P. Holland, Phys. Rev. A 96, 013413 (2017). 
D. M. P. Holland, I. Powis, A. B. Trofimov, R. C. Menzies, A. W. Potts, L. Karlsson, I. L. Badsyuk, T. E. Moskovskaya, E. V. Gromov, and J. Schirmer, J. Chem. Phys. 147, 164307 (2017).

A. W. Potts, D. M. P. Holland, I. Powis, L. Karlsson, A. B. Trofimov, and I. L. Bodzuk, Chem. Phys. 415, 84 (2013).

I. Powis, A. B. Trofimov, I. L. Bodzuk, D. M. P. Holland, A. W. Potts, and L. Karlsson, Chem. Phys 415, 291 (2013).

A. W. Potts, I. Novak, F. Quinn, G. V. Marr, B. Dobson, I. H. Hillier, and J. B. West, J. Phys. B: At. Mol. Phys. 18, 3177 (1985).

J. Cooper and R. N. Zare, J. Chem. Phys. 48, 942 (1968).

P. Baltzer, L. Karlsson, M. Lundqvist, and B. Wannberg, Rev. Sci. Instrum. 64, 2179 (1993).

J. Jauhiainen, A. Ausmees, A. Kivimäki, S. J. Osborne, A. N. Naves de Brito, S. Aksela, S. Svensson, and H. Aksela, J. Electron Spectrosc. Relat. Phenom. 69, 181 (1994).

I. Powis, D. M. P. Holland, E. Antonsson, M. Patanen, C. Nicolas, C. Miron, M. Schneider, D. Y. Soshnikov, A. Dreuw, and A. B. Trofimov, J. Chem. Phys. 143, 144304 (2015).

W. von Niessen, J. Schirmer, and L. S. Cederbaum, Comput. Phys. Rep. 1, 57 (1984).

V. G. Zakrzewski and J. V. Ortiz, Int. J. Quantum Chem. Suppl. 28, 23 (1994).

H. Sekino and R. J. Bartlett, Int. J. Quantum Chem. Suppl. 18, 255 (1984).

M. Nooijen and J. G. Snijders, Int. J. Quantum Chem. Suppl. 26, 55 (1992).

J. F. Stanton and J. Gauss, J. Chem. Phys. 103, 1064 (1995).

J. Schirmer, L. S. Cederbaum, and O. Walter, Phys. Rev. A 28, 1237 (1983).

J. Schirmer and G. Angonoa, J. Chem. Phys. 91, 1754 (1989).

M. J. Frisch, G. W. Trucks, H. B. Schlegel, G. E. Scuseria, M. A. Robb, J. R. Cheeseman, G. Scalmani, V. Barone, G. A. Petersson, H. Nakatsuji, X. Li, M. Caricato, A. V. Marenich, J. Bloino, B. G. Janesko, R. Gomperts, B. Mennucci, H. P. Hratchian, J. V. Ortiz, A. F. Izmaylov, J. L. Sonnenberg, D. Williams-Young, F. Ding, F. Lipparini, F. Egidi, J. Goings, B. Peng, A. Petrone, T. Henderson, D. Ranasinghe, V. G. Zakrzewski, J. Gao, N. Rega, G. Zheng, W. Liang, M. Hada, M. Ehara, K. Toyota, R. Fukuda, J. Hasegawa, M. Ishida, T. Nakajima, Y. Honda, O. Kitao, H. Nakai, T. Vreven, K. Throssell, J. J. A. Montgomery, J. E. Peralta, F. Ogliaro, M. Bearpark, J. J. Heyd, E. Brothers, K. N. Kudin, V. N. Staroverov, T. A. Keith, R. Kobayashi, J. Normand, K. Raghavachari, A. Rendell, J. C. Burant, S. S. Iyengar, J. Tomasi, M. Cossi, J. M. Millam, M. Klene, C. Adamo, R. Cammi, J. W. Ochterski, R. L. Martin, K. Morokuma, O. Farkas, J. B. Foresman, and D. J. Fox, Gaussian 16 Revision A.03 (Gaussian Inc., Wallingford, CT, 2016).

Y. Shao, Z. Gan, E. Epifanovsky, A. T. B. Gilbert, M. Wormit, J. Kussmann, A. W. Lange, A. Behn, J. Deng, X. Feng, D. Ghosh, M. Goldey, P. R. Horn, L. D. Jacobson, I. Kaliman, R. Z. Khaliullin, T. Kuś, A. Landau, J. Liu, E. I. Proynov, Y. M. Rhee, R. M. Richard, M. A. Rohrdanz, R. P. Steele, E. J. Sundstrom, H. L. Woodcock, P. M. Zimmerman, D. Zuev, B. Albrecht, E. Alguire, B. Austin, G. J. O. Beran, Y. A. Bernard, E. Berquist, K. Brandhorst, K. B. Bravaya, S. T. Brown, D. Casanova, C.-M. Chang, Y. Chen, S. H. Chien, K. D. Closser, D. L. Crittenden, M. Diedenhofen, R. A. DiStasio, H. Do, A. D. Dutoi, R. G. Edgar, S. Fatehi, L. Fusti-Molnar, A. Ghysels, A. GolubevaZadorozhnaya, J. Gomes, M. W. D. Hanson-Heine, P. H. P. Harbach, A. W. Hauser, E. G. Hohenstein, Z. C. Holden, T.-C. Jagau, H. Ji, B. Kaduk, K. Khistyaev, J. Kim, J. Kim, R. A. King, P. Klunzinger, D. Kosenkov, T. Kowalczyk, C. M. Krauter, K. U. Lao, A. D. Laurent, K. V. Lawler, S. V. Levchenko, C. Y. Lin, F. Liu, E. Livshits, R. C. Lochan, A. Luenser, P. Manohar, S. F. Manzer, S.-P. Mao, N. Mardirossian, A. V. Marenich, S. A. Maurer, N. J. Mayhall, E. Neuscamman, C. M. Oana, R. Olivares-Amaya, D. P. O'Neill, J. A. Parkhill, T. M. Perrine, R. Peverati, A. Prociuk, D. R. Rehn, E. Rosta, N. J. Russ, S. M. Sharada, S. Sharma, D. W. Small, A. Sodt, T. Stein, D. Stück, Y.-C. Su, A. J. W. Thom, T. Tsuchimochi, V. Vanovschi, L. Vogt, O. Vydrov, T. Wang, M. A. Watson, J. Wenzel, A. White, C. F. Williams, J. Yang, S. Yeganeh, S. R. Yost, Z.-Q. You, I. Y. Zhang, X. Zhang, Y. Zhao, B. R. Brooks, G. K. L. Chan, D. M. Chipman, C. J. Cramer, W. A. Goddard, M. S. Gordon, W. J. Hehre, A. Klamt, H. F. Schaefer, M. W. Schmidt, C. D. Sherrill, D. G. Truhlar, A. Warshel, X. Xu, A. Aspuru-Guzik, R. Baer, A. T. Bell, N. A. Besley, J.-D. Chai, A. Dreuw, B. D. 
Dunietz, T. R. Furlani, S. R. Gwaltney, C.-P. Hsu, Y. Jung, J. Kong, D. S. Lambrecht, W. Liang, C. Ochsenfeld, V. A. Rassolov, L. V. Slipchenko, J. E. Subotnik, T. Van Voorhis, J. M. Herbert, A. I. Krylov, P. M. W. Gill and M. Head-Gordon, Mol. Phys. 113, 184 (2015).

M. W. Schmidt, K. K. Baldridge, J. A. Boatz, S. T. Elbert, M. S. Gordon, J. H. Jensen, S. Koseki, N. Matsunaga, K. A. Nguyen, S. J. Su, T. L. Windus, M. Dupuis, and J. A. Montgomery, J. Comput. Chem. 14, 1347 (1993).

M. S. Gordon and M. W. Schmidt, in Theory and Applications of Computational Chemistry, edited by C. E. Dykstra, G. Frenking, K. S. Kim, and G. E. Scuseria (Elsevier, Amsterdam, 2005), pp. 1167.

D. E. Woon and T. H. Dunning, J. Chem. Phys. 98, 1358 (1993).

T. H. Dunning, J. Chem. Phys. 90, 1007 (1989).

L. Schafer, J. D. Ewbank, K. Siam, D. W. Paul, and D. L. Monts, J. Mol. Struct. 145, 135 (1986).

D. Dill and J. L. Dehmer, J. Chem. Phys. 61, 692 (1974).

J. W. Davenport, Dissertation, University of Pennsylvania, 1976.

P. Downie and I. Powis, J. Chem. Phys. 111, 4535 (1999).

M. Staniforth, S. Daly, K. L. Reid, and I. Powis, J. Chem. Phys. 139, 064304 (2013).

J. C. Slater, The self-consistent field for molecules and solids. (Mc-Graw Hill, New York, 1974).

L. S. Cederbaum, W. Domcke, J. Schirmer, and W. Von Niessen, Adv. Chem. Phys. 65, 115 (1986).

M. C. H. Wong, A. T. Le, A. F. Alharbi, A. E. Boguslavskiy, R. R. Lucchese, J. P. Brichta, C. D. Lin, and V. R. Bhardwaj, Phys. Rev. Lett. 110, 033006 (2013). E. R. Davidson and W. T. Borden, J. Phys. Chem. 87, 4783 (1983). (2004).

M. P. Andersson and P. Uvdal, J. Phys. Chem. A 109, 2937 (2005). 


\section{Tables}

\section{Table 1}

Binding energy regions used to define the electronic bands when extracting photoelectron anisotropy parameters and branching ratios from the polarization dependent spectra.

\begin{tabular}{|c|c|}
\hline Band & Binding energy range (eV) \\
\hline$X$ & $9.60-10.50$ \\
\hline$A$ & $11.80-12.00$ \\
\hline$B$ & $12.00-12.30$ \\
\hline$C$ & $12.55-13.10$ \\
\hline$D$ & $13.50-14.00$ \\
\hline$E$ & $14.00-15.00$ \\
\hline$F / G$ & $15.60-17.40$ \\
\hline
\end{tabular}




\section{Table 2.}

Energies $E(\mathrm{eV})$ and intensities $P$ of the outer valence vertical ionization transitions in transdichloroethene computed using the HF, OVGF, ADC(3), and EOM-IP-CCSD methods and the cc-pVTZ basis set. The experimental values are given for comparison. Only transitions with $P \geq 0.01$ are shown.

\begin{tabular}{|c|c|c|c|c|c|c|c|c|c|c|}
\hline \multirow{2}{*}{$\begin{array}{l}\text { Cationic } \\
\text { state }\end{array}$} & \multirow{2}{*}{$\begin{array}{c}\text { Molecular } \\
\text { orbital }\end{array}$} & \multirow[t]{2}{*}{ Type } & \multirow{2}{*}{$\begin{array}{c}\mathrm{HF} \\
E\end{array}$} & \multicolumn{2}{|c|}{ OVGF } & \multicolumn{2}{|c|}{$\operatorname{ADC}(3)$} & \multicolumn{2}{|c|}{$\begin{array}{c}\text { EOM-IP- } \\
\text { CCSD }\end{array}$} & \multirow{2}{*}{$\frac{\text { Expt. }^{\mathrm{a}}}{E}$} \\
\hline & & & & $E$ & $P$ & $E$ & $P$ & $E$ & $P^{\mathrm{b}}$ & \\
\hline$x^{2} A_{u}$ & $3 a_{u}$ & $\pi$ & 9.93 & 9.68 & 0.91 & 9.72 & 0.90 & 9.76 & 0.92 & $9.8^{c}$ \\
\hline$A^{2} \mathrm{~A}_{\mathrm{g}}$ & $10 a_{g}$ & $\sigma_{\mathrm{Cl} L P}$ & 12.67 & 11.86 & 0.90 & 11.84 & 0.89 & 11.88 & 0.93 & \multirow{2}{*}{$11.8^{c}$} \\
\hline$B^{2} B_{u}$ & $9 b_{u}$ & $\sigma_{C l L P}$ & 12.83 & 12.04 & 0.90 & 11.98 & 0.89 & 12.04 & 0.93 & \\
\hline$C^{2} \mathrm{~B}_{\mathrm{g}}$ & $2 b_{g}$ & $\pi_{\mathrm{CI} L \mathrm{P}}$ & 13.53 & 12.70 & 0.90 & 12.64 & 0.87 & 12.71 & 0.93 & $12.6^{c}$ \\
\hline$D^{2} \mathrm{~A}_{\mathrm{u}}$ & $2 a_{u}$ & $\pi$ & 14.91 & 13.88 & 0.88 & 13.93 & 0.85 & 13.96 & 0.92 & 13.8 \\
\hline$E^{2} \mathrm{~A}_{\mathrm{g}}$ & $9 a_{g}$ & $\sigma$ & 14.98 & 14.05 & 0.90 & 14.15 & 0.89 & 14.16 & 0.93 & 14.2 \\
\hline${ }^{2} B_{g}{ }^{d}$ & & & & & & 14.45 & 0.01 & & & \\
\hline$F^{2} \mathrm{~B}_{u}{ }^{e}$ & $8 b_{u}$ & $\sigma$ & 17.67 & 16.43 & 0.87 & 16.45 & 0.82 & 16.53 & 0.91 & \multirow{2}{*}{16.2} \\
\hline$G^{2} \mathrm{Ag}_{\mathrm{g}}^{\mathrm{e}}$ & $8 a_{g}$ & $\sigma$ & 17.90 & 16.26 & 0.89 & 16.49 & 0.88 & 16.36 & 0.92 & \\
\hline
\end{tabular}

a Band maxima, as estimated from the present photoelectron spectrum recorded at a photon energy of $80 \mathrm{eV}$.

${ }^{b}$ The EOM-IP-CCSD pole strength is evaluated as one-hole character of the final state wavefunction.

c The lowest energy peak in these bands, assumed to be near to the adiabatic transitions, occur at 9.631, 11.843 and $12.589 \mathrm{eV}$ for the $X^{2} \mathrm{~A}_{\mathrm{u}}, A^{2} \mathrm{~A}_{\mathrm{g}} / B^{2} \mathrm{~B}_{\mathrm{u}}$ and $C^{2} \mathrm{~B}_{\mathrm{g}}$ states, respectively.

d $3 a_{u}{ }^{-2} b_{g}$ two-electron excitation/ionization

e The $F$ and $G$ band identification shown here as, respectively, $\left(8 b_{u}\right)^{-1}$ and $\left(8 a_{g}\right)^{-1}$ ionizations are based upon the HF orbital ordering. The other calculations shown here indicate the reverse ordering should be applied. 


\section{Table 3.}

Mulliken atomic population in the outer valence molecular orbitals of trans-dichloroethene (units are electrons; sum over all atoms is 2) calculated at the HF/cc-pVTZ level; the $\sigma, \pi$ orbital descriptions are used to indicate, respectively, the in- or out-of plane character of the orbital.

\begin{tabular}{|c|c|c|c|c|c|c|c|c|}
\hline Atom & $\begin{array}{c}8 \mathrm{a}_{\mathrm{g}} \\
(\sigma)\end{array}$ & $\begin{array}{c}8 \mathrm{~b}_{\mathrm{u}} \\
(\sigma)\end{array}$ & $\begin{array}{c}9 \mathrm{a}_{\mathrm{g}} \\
(\sigma)\end{array}$ & $\begin{array}{c}2 \mathrm{a}_{\mathrm{u}} \\
(\pi)\end{array}$ & $\begin{array}{c}2 \mathrm{~b}_{\mathrm{g}} \\
\left(\pi_{\mathrm{CL} L \mathrm{LP}}\right)\end{array}$ & $\begin{array}{c}9 \mathrm{~b}_{\mathrm{u}} \\
\left(\sigma_{\mathrm{CILP}}\right)\end{array}$ & $\begin{array}{c}10 \mathrm{a}_{\mathrm{g}} \\
\left(\sigma_{\mathrm{ClLP}}\right)\end{array}$ & $\begin{array}{c}3 \mathrm{a}_{\mathrm{u}} \\
(\pi)\end{array}$ \\
\hline $\mathrm{C}$ & 0.59 & 0.36 & 0.32 & 0.41 & 0.05 & 0.03 & 0.07 & 0.57 \\
\hline $\mathrm{H}$ & 0.22 & 0.06 & 0.12 & 0.0 & 0.0 & 0.02 & 0.04 & 0.01 \\
\hline $\mathrm{Cl}$ & 0.19 & 0.58 & 0.56 & 0.58 & 0.95 & 0.94 & 0.89 & 0.42 \\
\hline \hline
\end{tabular}




\section{Table 4.}

Intrastate coupling constants $\kappa(\mathrm{eV})$ and Poisson parameters $a$ for the five lowest excited states of the trans-dichloroethene radical cation along the totally symmetric $\left(a_{\mathrm{g}}\right)$ vibrational modes; maximal vibrational quantum numbers $n_{s}$ for individual modes used in the direct product basis for the $\left(A^{2} A_{g}, B\right.$ $\left.{ }^{2} \mathrm{~B}_{u}, C^{2} \mathrm{~B}_{\mathrm{g}}\right)$ and $\left(D^{2} \mathrm{~A}_{u}, E^{2} \mathrm{~A}_{\mathrm{g}}\right)$ spectra computations.

\begin{tabular}{|c|c|c|c|c|c|c|c|}
\hline Mode & $A^{2} \mathrm{~A}_{\mathrm{g}}$ & $B^{2} \mathrm{~B}_{\mathrm{u}}$ & $C^{2} \mathrm{~B}_{\mathrm{g}}$ & $n_{s}(A-B-C)$ & $D^{2} \mathrm{~A}_{\mathrm{u}}$ & $E^{2} \mathrm{~A}_{\mathrm{g}}$ & $n_{s}(E-D)$ \\
\hline$\kappa$ & & & & & & & \\
\hline$v_{1}{ }^{+}$ & 0.017 & 0.042 & 0.005 & & -0.054 & 0.180 & \\
\hline$v_{2}{ }^{+}$ & -0.050 & 0.032 & 0.044 & & -0.062 & 0.170 & \\
\hline$v_{3}{ }^{+}$ & -0.036 & -0.049 & -0.045 & & -0.046 & -0.105 & \\
\hline$v_{4}{ }^{+}$ & -0.034 & 0.014 & 0.082 & & 0.089 & 0.080 & \\
\hline$v_{5}{ }^{+}$ & -0.009 & -0.018 & 0.012 & & 0.060 & 0.033 & \\
\hline$a$ & & & & & & & \\
\hline$v_{1}{ }^{+}$ & 0.002 & 0.011 & 0.0 & - & 0.018 & 0.199 & 4 \\
\hline$v_{2}{ }^{+}$ & 0.061 & 0.026 & 0.046 & 2 & 0.094 & 0.699 & 10 \\
\hline$v_{3}{ }^{+}$ & 0.051 & 0.091 & 0.078 & 4 & 0.081 & 0.423 & 8 \\
\hline$v_{4}{ }^{+}$ & 0.099 & 0.015 & 0.568 & 8 & 0.668 & 0.546 & 10 \\
\hline$v_{5}{ }^{+}$ & 0.042 & 0.155 & 0.069 & 4 & 1.822 & 0.556 & 20 \\
\hline \hline
\end{tabular}




\section{Table 5}

Interstate coupling constants $\lambda(\mathrm{eV})$ and Poisson parameters $a$ within the two groups of cationic states $\left(A{ }^{2} \mathrm{~A}_{\mathrm{g}}, B{ }^{2} \mathrm{~B}_{\mathrm{u}}, C^{2} \mathrm{~B}_{\mathrm{g}}\right)$ and $\left(D^{2} \mathrm{~A}_{\mathrm{u}}, E^{2} \mathrm{~A}_{\mathrm{g}}\right)$, treated in the present linear vibronic coupling models; maximal vibrational quantum numbers $n_{s}$ for individual modes used in the direct product basis for the $\left(A^{2} \mathrm{~A}_{\mathrm{g}}, B^{2} \mathrm{~B}_{\mathrm{u}}, C^{2} \mathrm{~B}_{\mathrm{g}}\right)$ and $\left(D^{2} \mathrm{~A}_{\mathrm{u}}, E^{2} \mathrm{~A}_{\mathrm{g}}\right)$ spectra computations.

\begin{tabular}{|c|c|c|c|c|c|c|c|c|c|}
\hline \multirow{2}{*}{ Constant } & \multicolumn{4}{|c|}{$A^{2} \mathrm{~A}_{\mathrm{g}}-B^{2} \mathrm{~B}_{\mathrm{u}}$} & $A^{2} \mathrm{~A}_{g}-C^{2} \mathrm{~B}_{\mathrm{g}}$ & \multicolumn{2}{|c|}{$B^{2} \mathrm{~B}_{\mathrm{u}}-C^{2} \mathrm{~B}_{\mathrm{g}}$} & \multicolumn{2}{|c|}{$D^{2} \mathrm{~A}_{\mathrm{u}}-E^{2} \mathrm{~A}_{\mathrm{g}}$} \\
\hline & $v_{9}^{+}\left(b_{\mathrm{u}}\right)$ & $v_{10}{ }^{+}\left(b_{\mathrm{u}}\right)$ & $v_{11}^{+}\left(b_{\mathrm{u}}\right)$ & $v_{12}{ }^{+}\left(b_{\mathrm{u}}\right)$ & $v_{8}^{+}\left(b_{\mathrm{g}}\right)$ & $v_{6}{ }^{+}\left(a_{\mathrm{u}}\right)$ & $v_{7}^{+}\left(a_{u}\right)$ & $v_{6}^{+}\left(a_{\mathrm{u}}\right)$ & $v_{7}^{+}\left(a_{\mathrm{u}}\right)$ \\
\hline$\lambda$ & 0.014 & 0.036 & 0.035 & $-{ }^{a}$ & 0.034 & 0.038 & $-{ }^{a}$ & 0.118 & $-{ }^{a}$ \\
\hline$a$ & 0.001 & 0.055 & 0.105 & -0.952 & 0.120 & 0.109 & -1.541 & 1.025 & -1.790 \\
\hline$n_{s}$ & - & 6 & 10 & - & 10 & 10 & - & 20 & - \\
\hline
\end{tabular}

a The constant $\lambda$ cannot be determined since the potential energy surfaces along this mode are not described by the present linear vibronic coupling model. The Poisson parameter a (unphysically negative in this case) is shown to at least demonstrate the magnitude of the energy change along the respective coordinate (see text for details). 


\section{Table 6}

Calculated (OVGF/cc-pVTZ, LVC model) vertical $\left(E_{v}\right)$ and adiabatic $\left(E_{0-0}\right)$ energies for the six lowest ionization transitions in trans-dichloroethene. $E_{\text {int }}$ is the minimal energy of the conical intersection between the potential energy surfaces for the two groups of cationic states, $\left(A^{2} \mathrm{~A}_{\mathrm{g}}, B^{2} \mathrm{~B}_{\mathrm{u}}, C^{2} \mathrm{~B}_{\mathrm{g}}\right)$ and $(D$ ${ }^{2} \mathrm{~A}_{u}, E^{2} \mathrm{~A}_{\mathrm{g}}$ ), treated in the present vibronic coupling models. All values are in eV.

\begin{tabular}{|c|c|c|c|c|c|}
\hline \hline & & & \multicolumn{3}{|c|}{$E_{\text {int }}{ }^{\mathrm{a}}$} \\
\hline State & $E_{v}$ & $E_{0-0}$ & $A^{2} \mathrm{~A}_{\mathrm{g}}$ & $B^{2} \mathrm{~B}_{\mathrm{u}}$ & $D^{2} \mathrm{~A}_{\mathrm{u}}$ \\
\hline$X^{2} \mathrm{~A}_{\mathrm{u}}$ & 9.68 & 9.40 & & & \\
\hline$A^{2} \mathrm{~A}_{\mathrm{g}}$ & 11.86 & 11.83 & & & \\
\hline$B^{2} \mathrm{~B}_{\mathrm{u}}$ & 12.04 & 12.01 & 12.07 & & \\
\hline$C^{2} \mathrm{~B}_{\mathrm{g}}$ & 12.70 & 12.61 & 13.12 & 13.70 & \\
\hline$D^{2} \mathrm{~A}_{\mathrm{u}}$ & 13.88 & $13.69^{\mathrm{b}}$ & & & \\
\hline$E^{2} \mathrm{~A}_{\mathrm{g}}$ & 14.05 & 13.68 & & & 13.80 \\
\hline \hline
\end{tabular}

a Obtained from the respective LVC models for pairs of interacting states.

b Saddle point. The present LVC model predicts a double-minimum potential energy surface, characterized by a stabilization energy of $0.017 \mathrm{eV}$. 
Figures

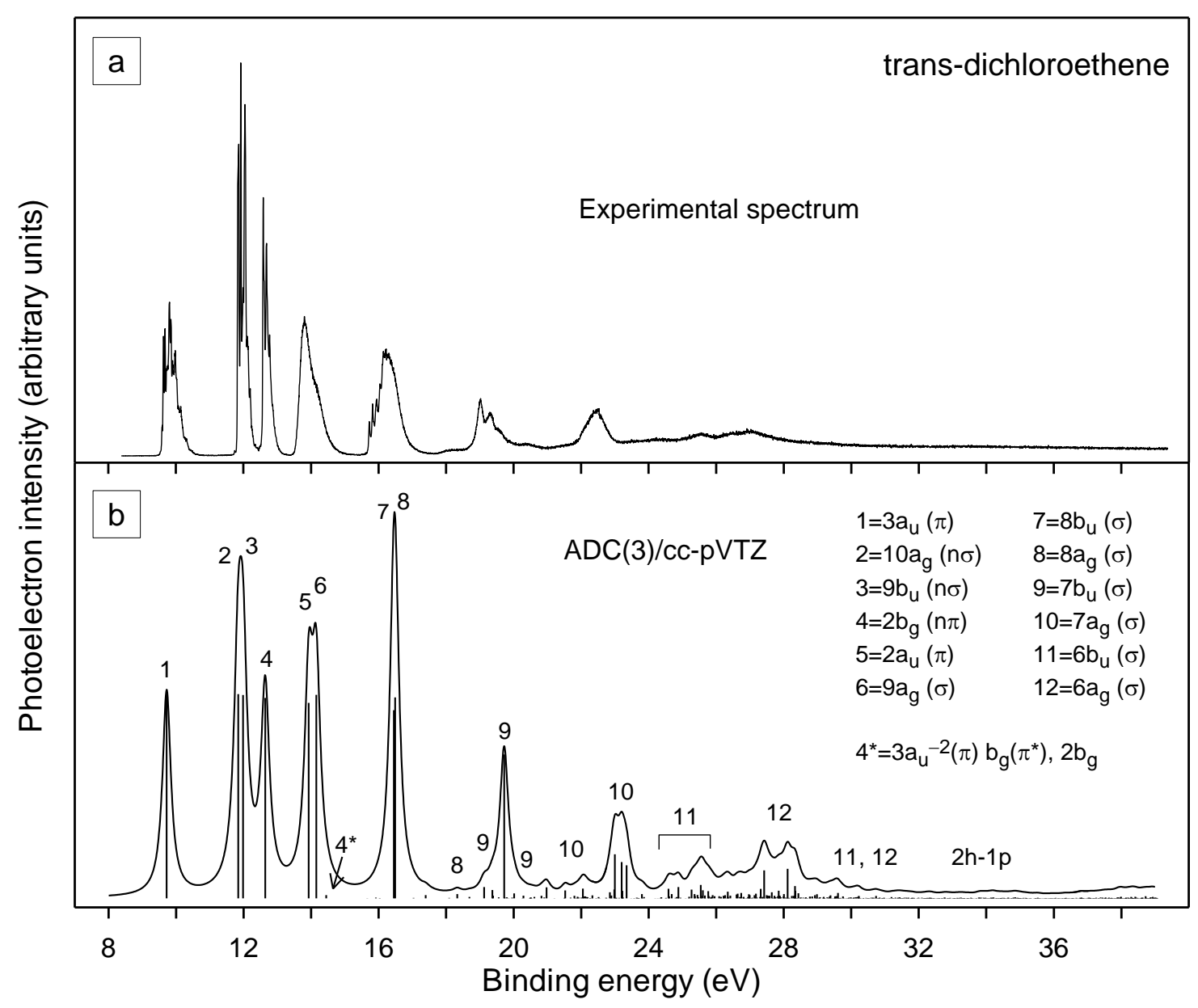

Figure 1

The valence shell photoelectron spectrum of trans-dichloroethene: (a) experimental spectrum recorded at a photon energy of $80 \mathrm{eV}$; (b) theoretical spectrum obtained using the $A D C(3)$ method and the cc-pVTZ basis set. 


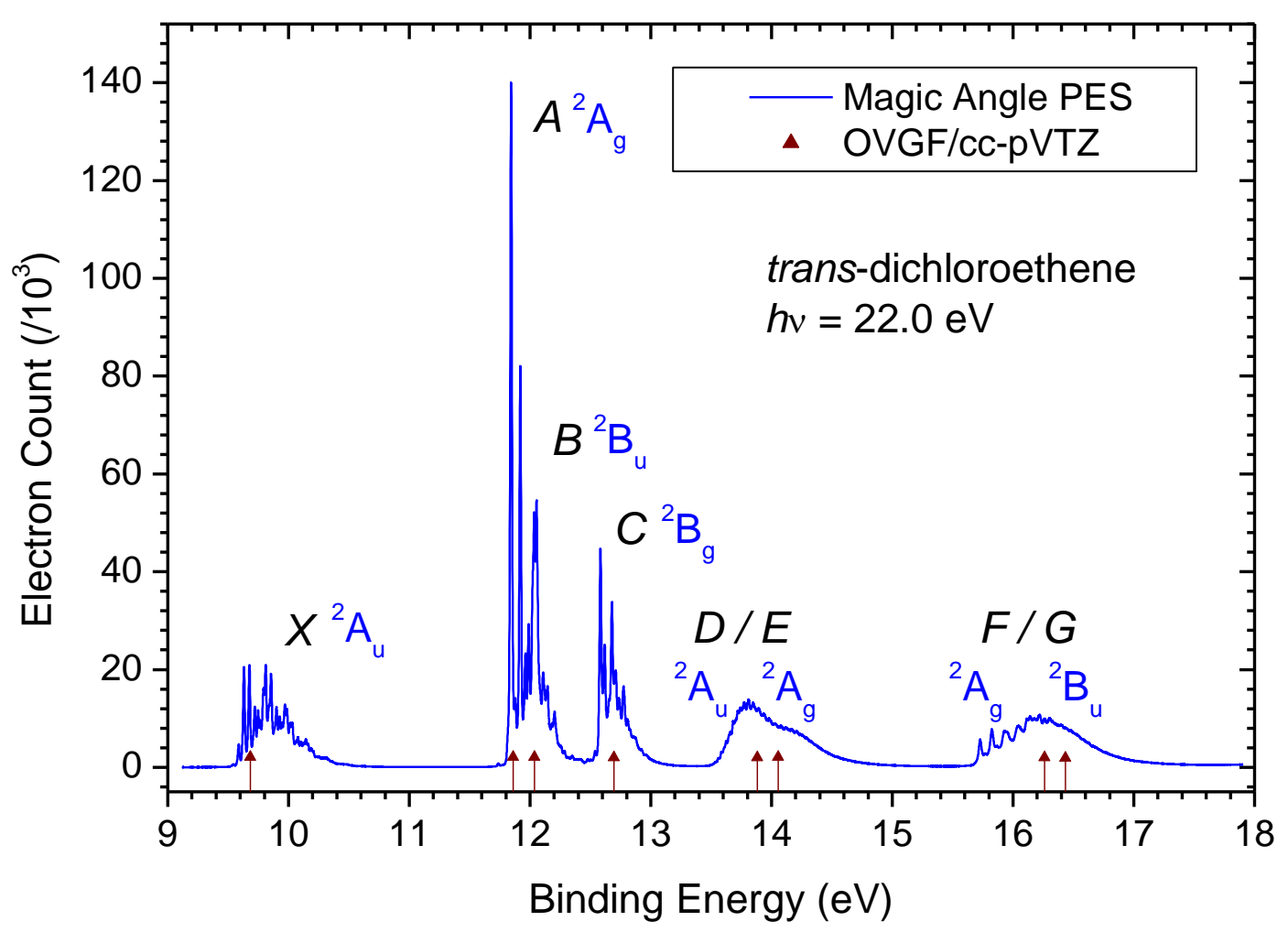

\section{Figure 2}

The outer valence region, Magic Angle, photoelectron spectrum of trans-dichloroethene, recorded with a photon energy of $22 \mathrm{eV}$. OVGF calculations of the vertical ionization energies are marked along the bottom. Note that, as shown in the figure and Table 2, these calculations reverse the energetic ordering of the $\left(8 \mathrm{ag}_{\mathrm{g}}\right)^{-1}$ and $\left(8 \mathrm{~b}_{\mathrm{u}}\right)^{-1}$ ionization relative to the $\mathrm{HF}$ orbital ordering. 


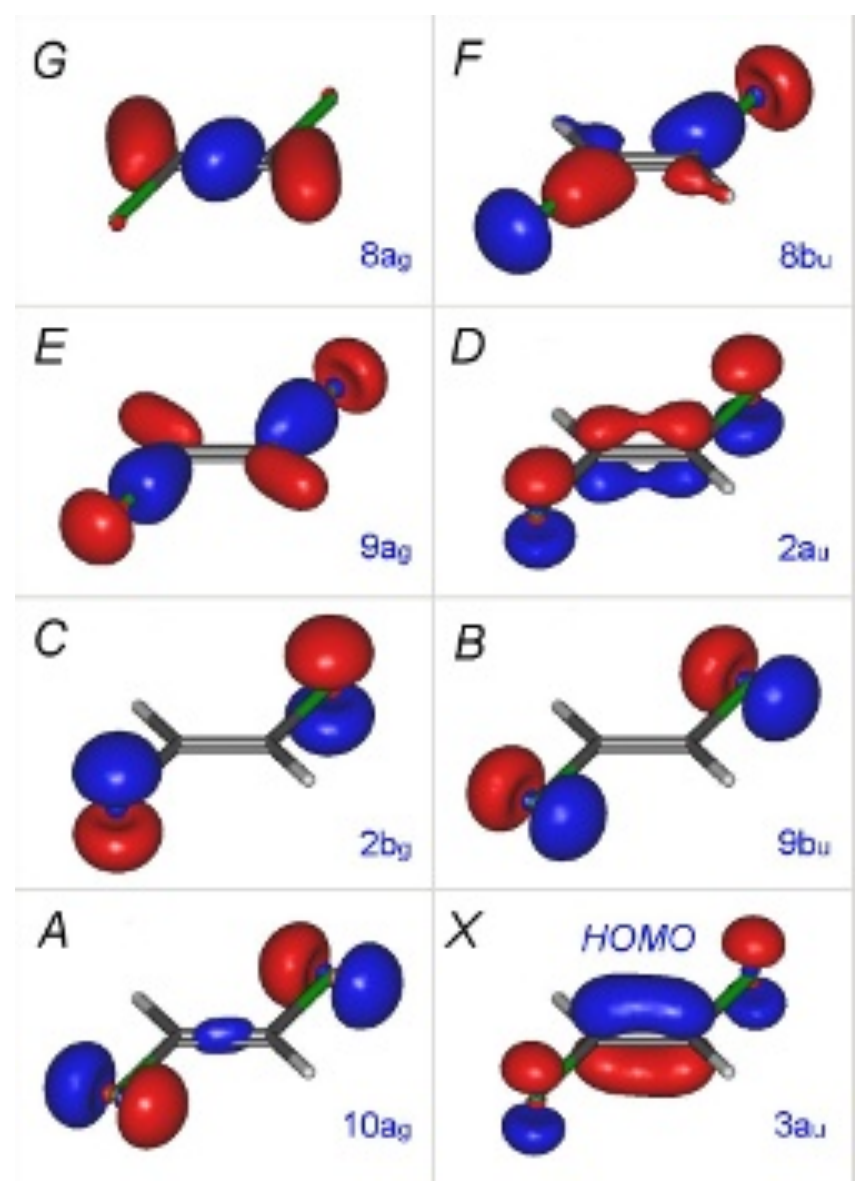

Figure 3

Iso-surface density plots of the eight highest occupied molecular orbitals of trans-dichloroethene produced using the results of the HF/cc-pVTZ calculations. 


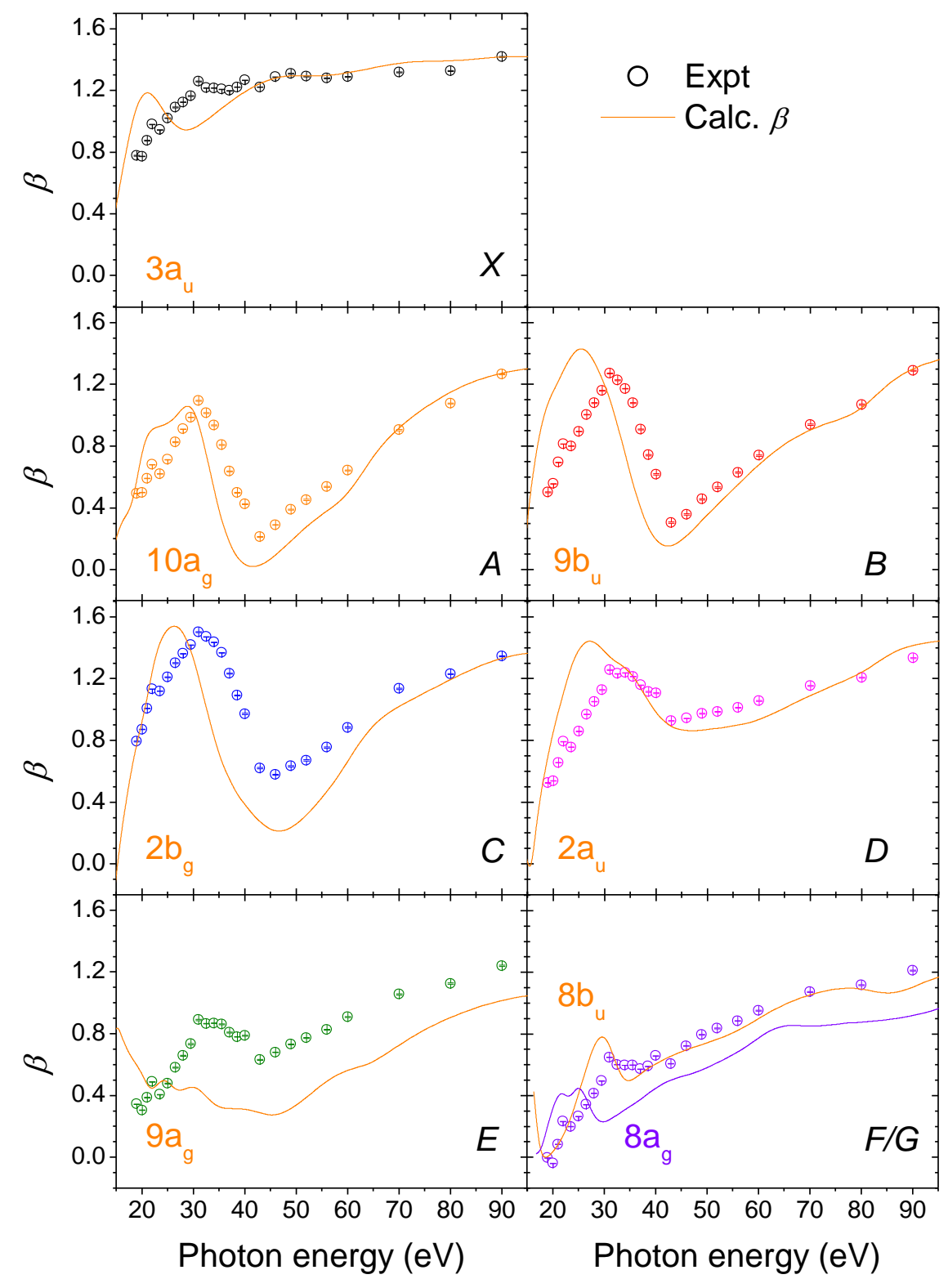

Figure 4

Photoelectron anisotropy parameters, $\beta$, measured for the outer valence states of transdichloroethene. Error bars on the experimental data points are typically no bigger than the plotting symbols. The $D / E$ bands overlap so the experimental division into $D$ and $E$ regions is somewhat arbitrary. CMS-X $\alpha$ calculations of $\beta$ for the corresponding orbital ionizations are shown for comparison. 


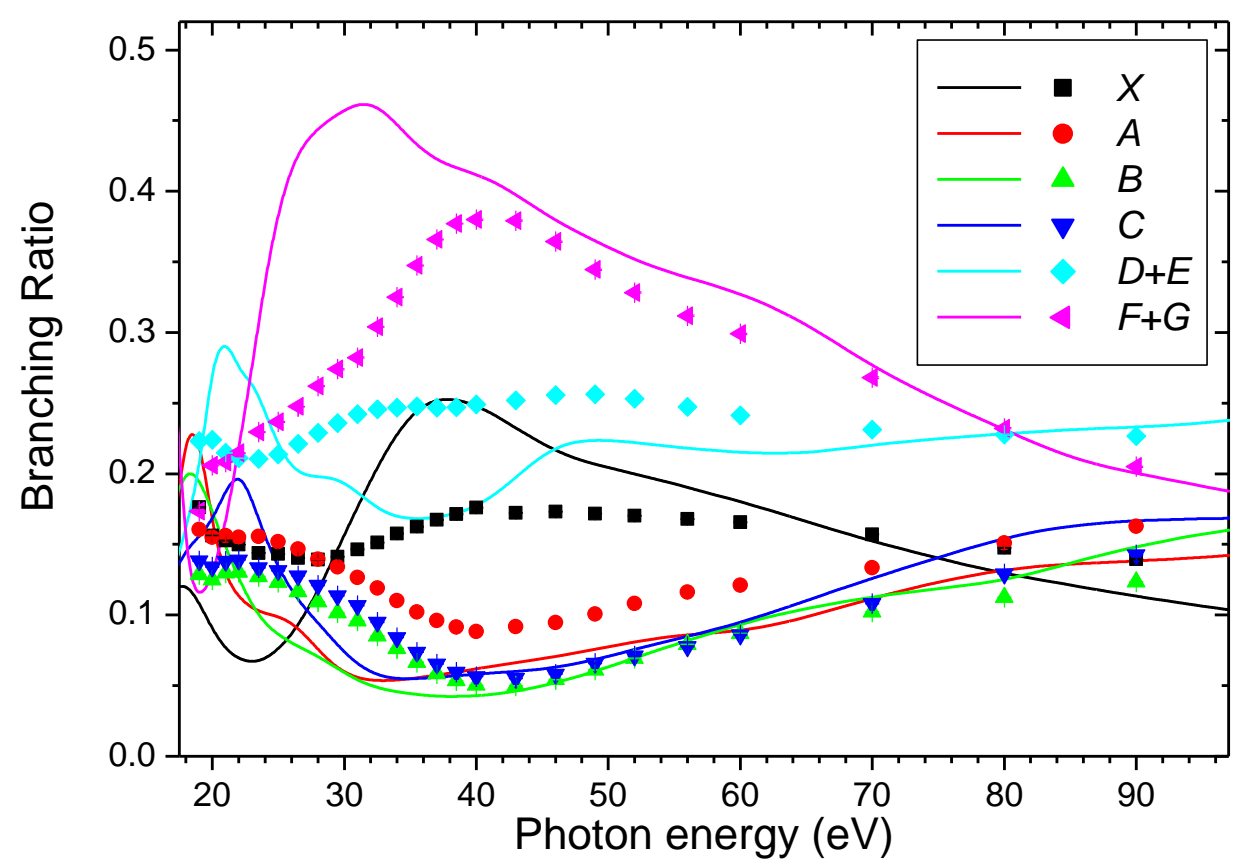

Figure 5

Branching ratios, obtained as normalized relative experimental band intensities (symbols) or calculated cross-section ratios (continuous curves). Error bars on the experimental data are typically no bigger than the plotting symbols. Because the experiments do not fully resolve the $D / E$ and $F / G$ bands, these data pairs are summed for plotting. 


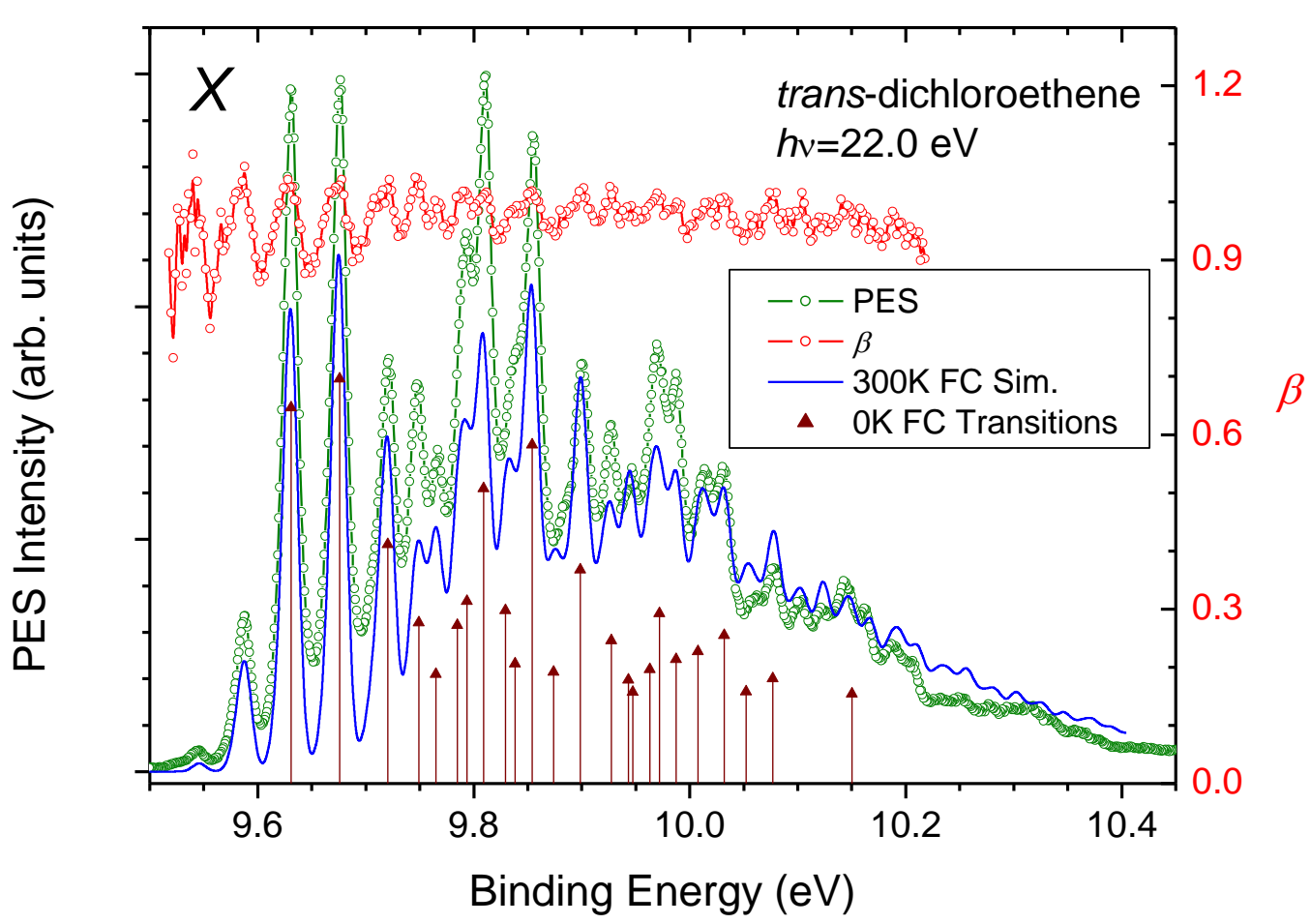

Figure 6

The $X$ band magic angle PES and Franck-Condon simulations, both arbitrarily scaled for best comparison. The FC simulation uses MP2/cc-pVTZ harmonic frequencies, scaled by a factor $0.96 .{ }^{69}$ Individual FC factors and positions are marked for $\mathrm{OK}$ transitions from the vibrationless ground state only, and for clarity this plotting has been restricted to the 25 most intense transitions. All calculated transitions are included in the $300 \mathrm{~K}$ simulation of the band profile. This was produced by folding the FC stick spectrum, including hot bands originating from thermally excited neutral levels, with a 7.5 meV HWHM Gaussian function to take account of experimental resolution and rotational widths. The spectrum of experimental $\beta$ parameters is also shown. 


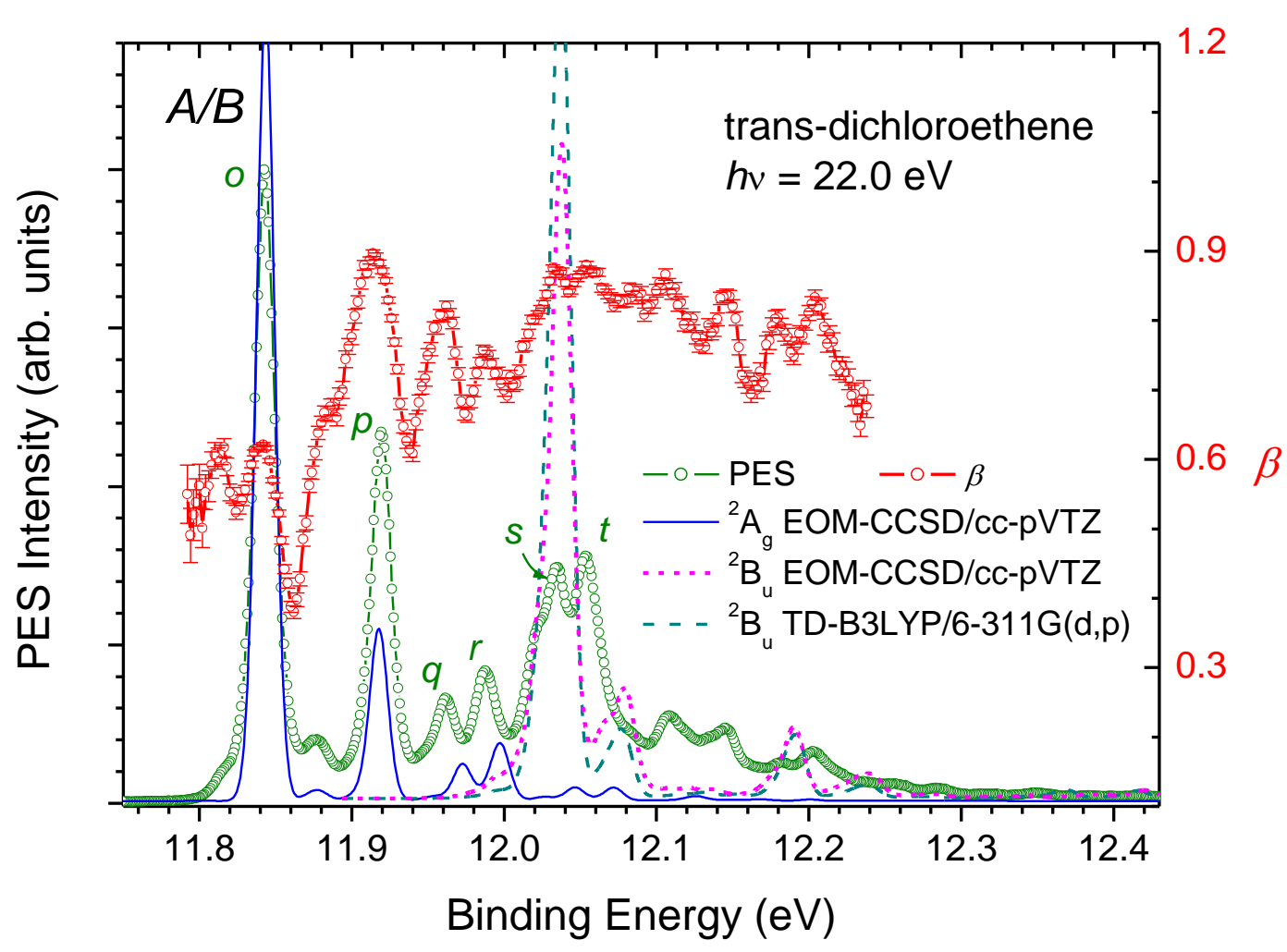

Figure 7

The $A / B$ band region of the magic-angle PES, corresponding $\beta$-parameter curve, and 300K FrankCondon simulations. The latter are arbitrarily scaled for comparison and the position of the $A{ }^{2} A_{g}$ simulation vibrational origin has been set at $11.843 \mathrm{eV}$ binding energy, as judged by eye. Vibrational origins of the $B{ }^{2} B_{u}$ simulations are positioned at the calculated EOM-IP-CCSD vertical ionization energy, $12.04 \mathrm{eV}$ (Table 2). All the FC simulations utilise a 7.5 meV HWHM Gaussian shaping function to represent contributions from experimental resolution and rotational widths. The principal experimental peaks are labelled $o-t$ for convenience when these are discussed in the text. 


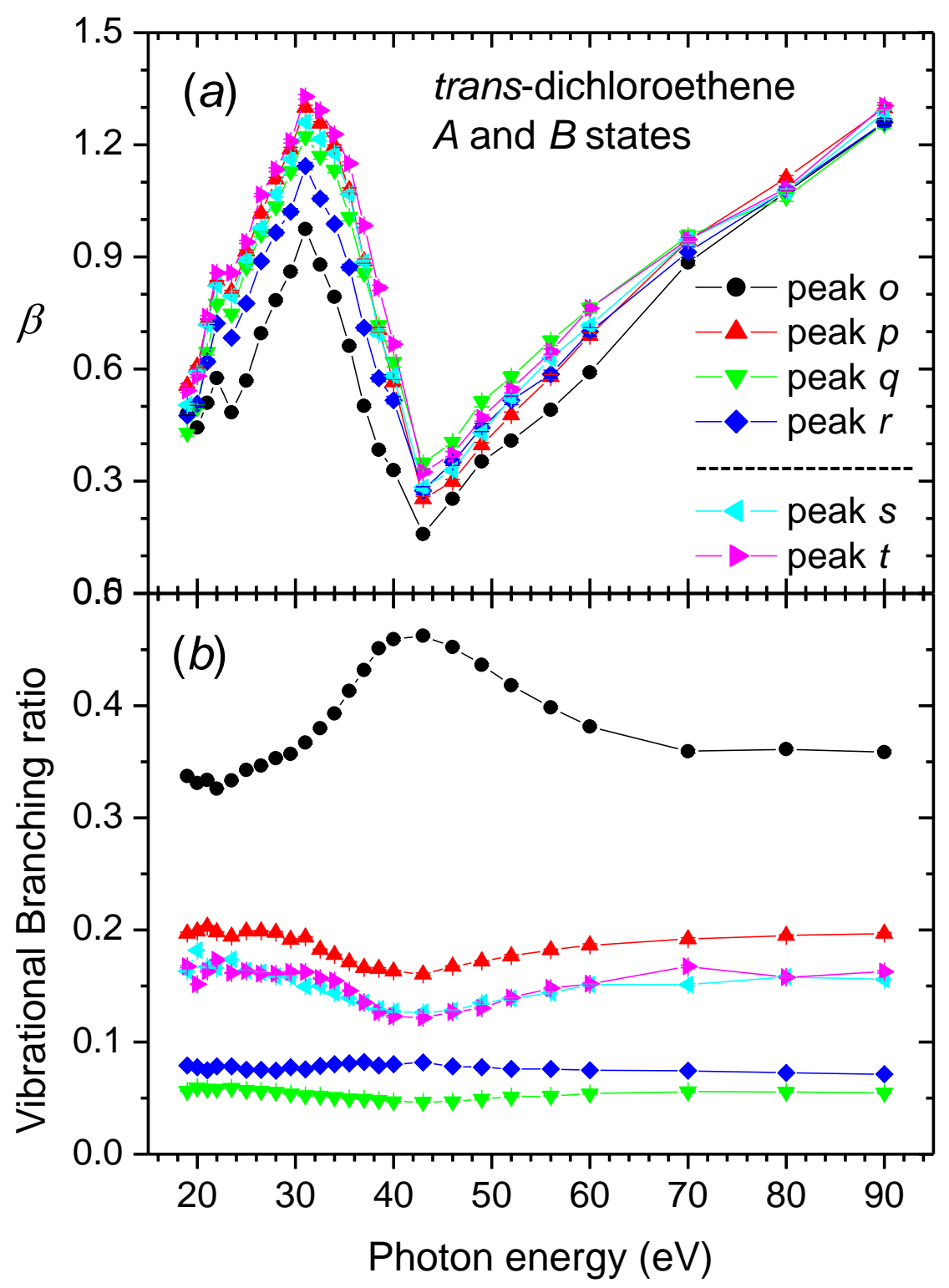

\section{Figure 8}

Vibrationally resolved $\beta$-parameters (a) and branching ratios (b) measured across the photon energy range of this study. Error bars on the individual data points are typically no bigger than the plotting symbols. The individual peaks are identified by labels $o-t$ as introduced in Fig. 7. 


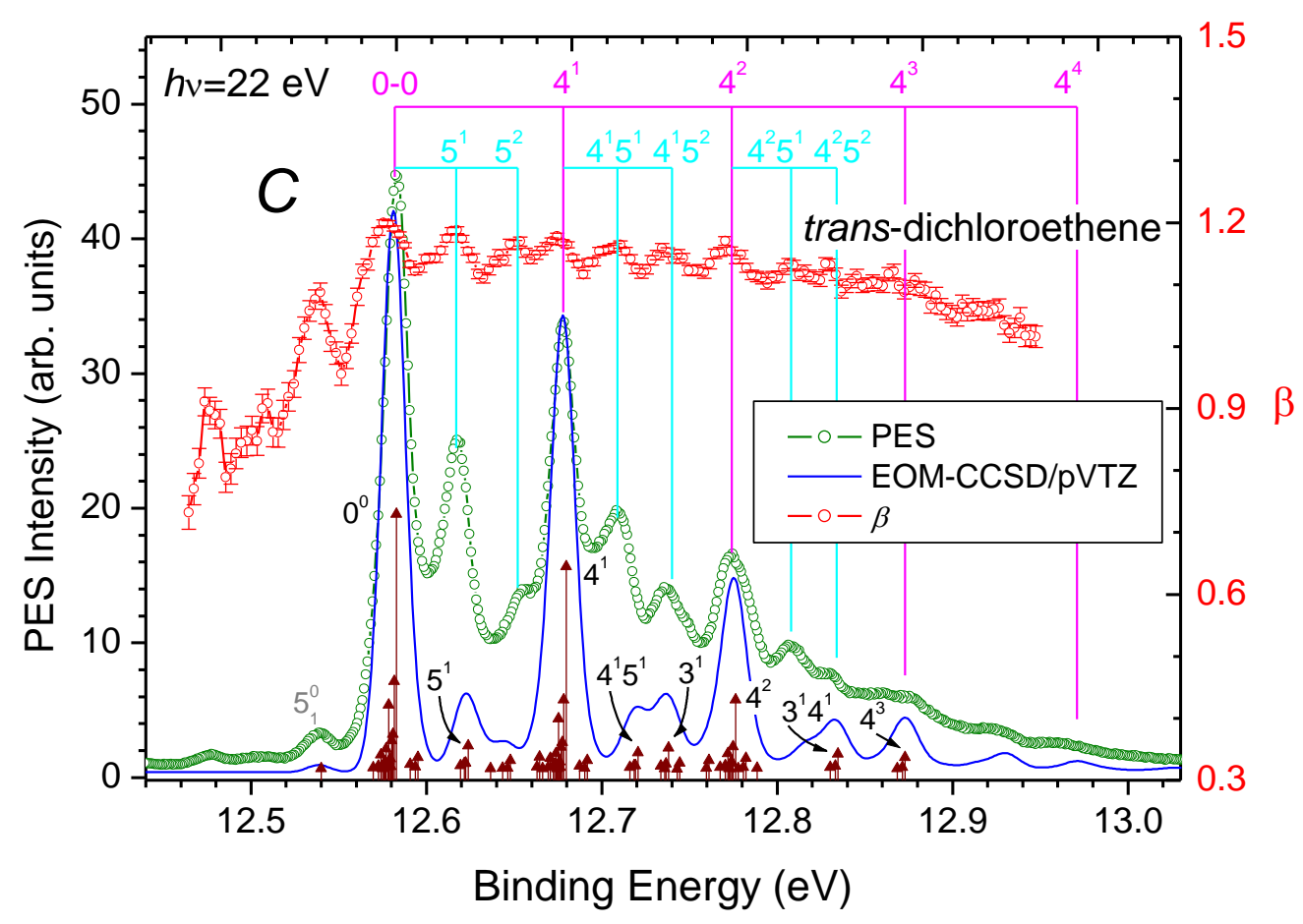

Figure 9

The $C$ band region of the PES and the corresponding $\beta$-parameter curve. A tentative assignment to progressions in the $v_{4} a_{\mathrm{g}}$ and $v_{5} a_{\mathrm{g}} \mathrm{Cl}$ stretch/bending modes is indicated along the top of the figure. A 300K Frank-Condon simulation, using EOM-CCSD/cc-pVTZ harmonic frequencies scaled by a factor 0.97, is also shown. For clarity only the 80 most intense FC factors are plotted as a stick spectrum but the full set has been convoluted with a $7.5 \mathrm{meV}$ HWHM Gaussian function to produce a more realistic impression that takes account of experimental resolution and rotational widths. The figure also includes the experimental $\beta$ parameter spectrum. 


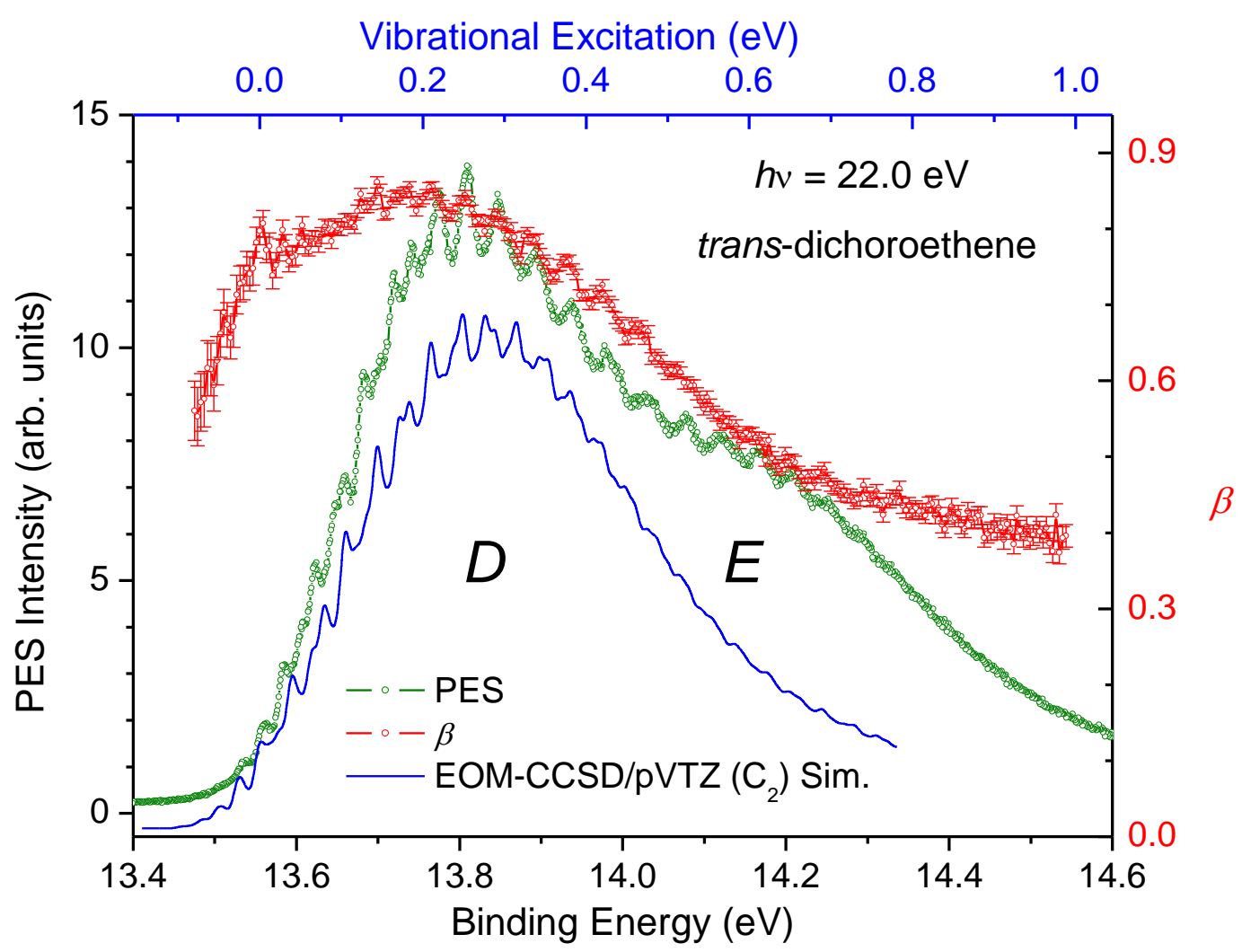

Figure 10

$D / E$ band region of the PES and the corresponding experimental $\beta$-parameter values. A 300K FC EOMCCSD/cc-pVTZ vibrational simulation for the twisted $\left(C_{2}\right)$ equilibrium geometry of the $D^{2} A_{u}$ state ion is plotted with arbitrary intensity, and the offset between the internal vibrational energy in the simulation (upper axis) and the experimental binding energy axis is judged by eye. 


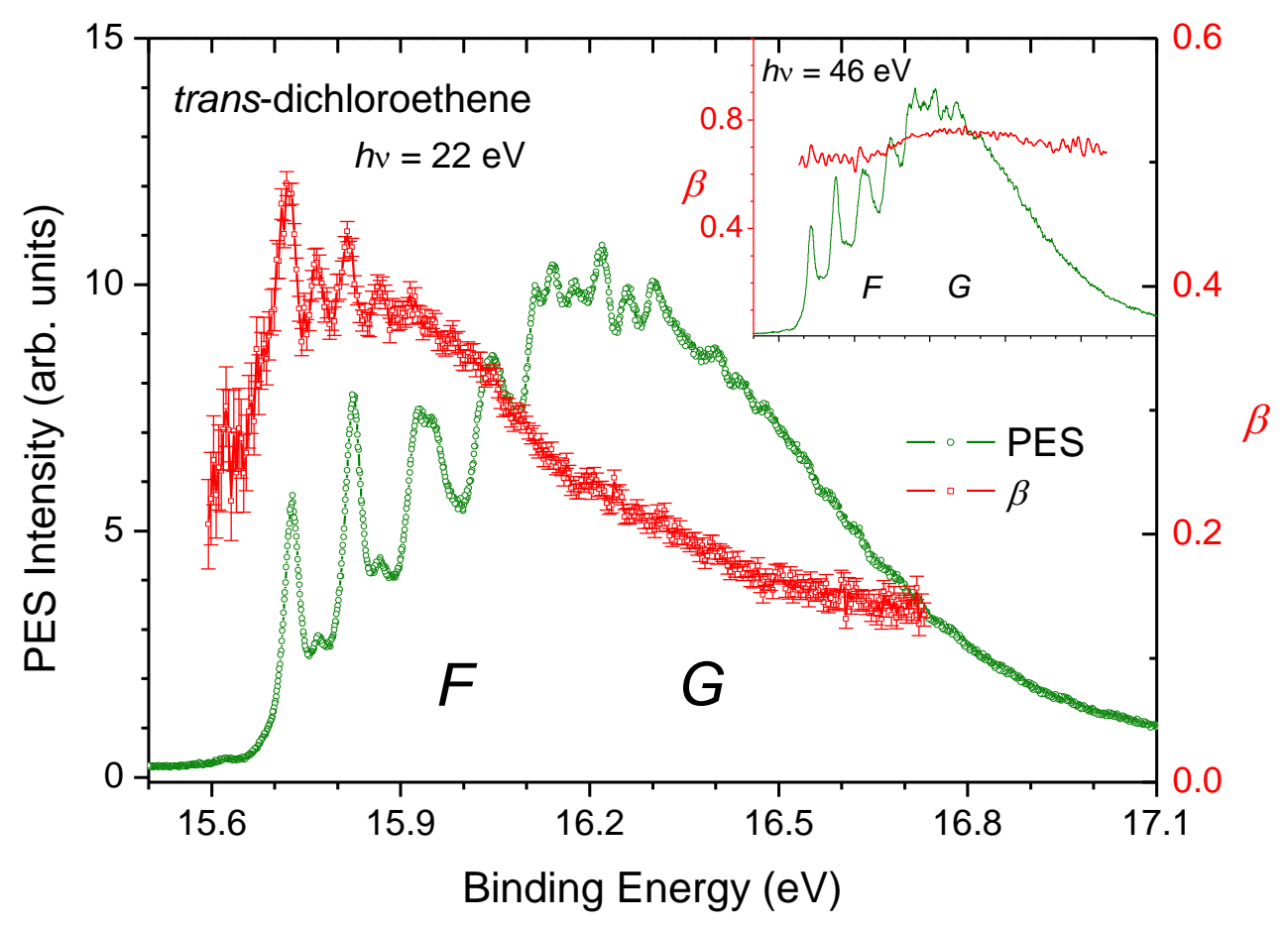

Figure 11

The $F / G$ band region of the PES and the corresponding $\beta$-parameter values recorded at a $22 \mathrm{eV}$ photon energy. The inset shows a similar data set recorded at $46 \mathrm{eV}$ photon energy. 


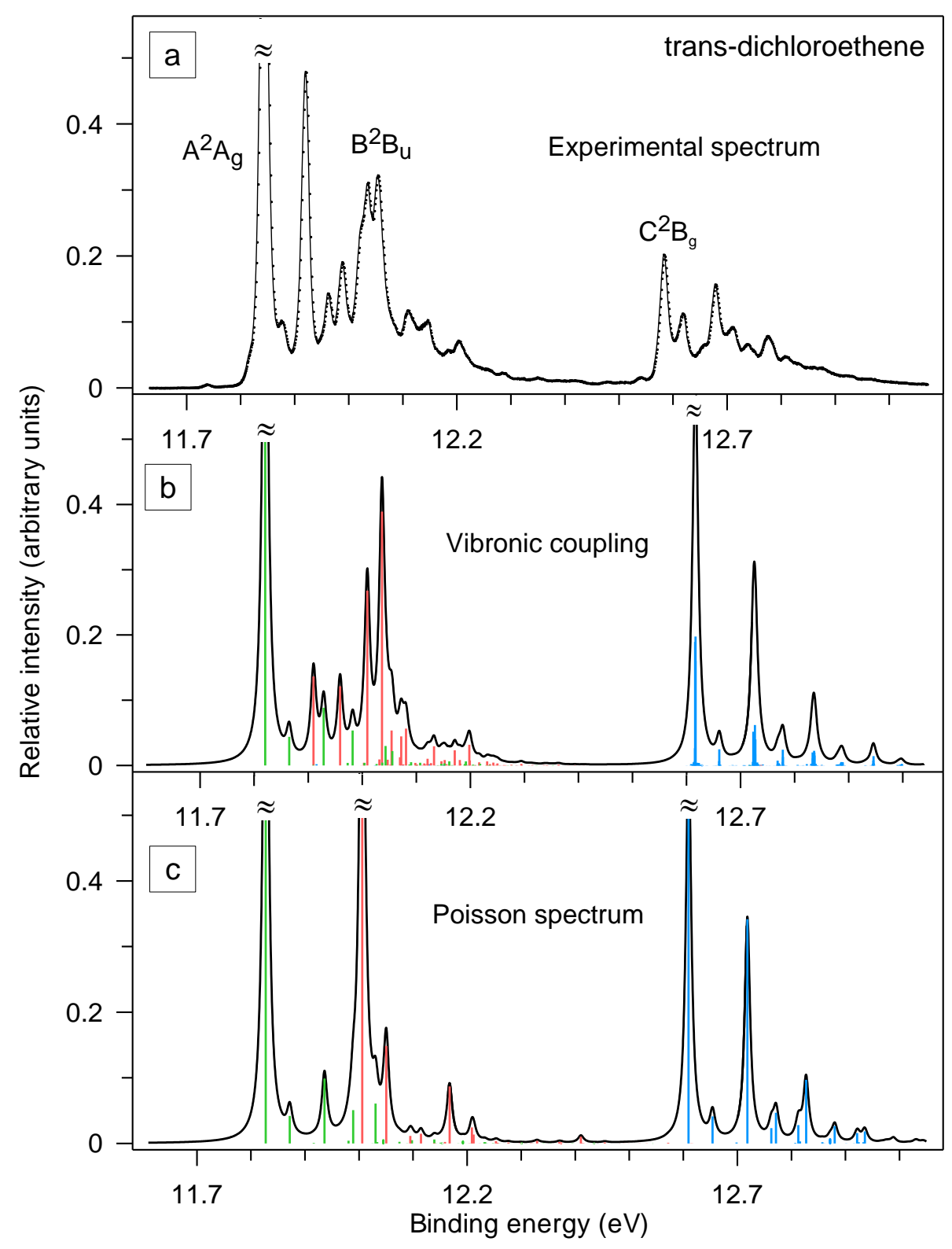

Figure 12

The $A{ }^{2} \mathrm{~A}_{\mathrm{g}}, B{ }^{2} \mathrm{~B}_{\mathrm{u}}$ and $C^{2}{ }^{2} \mathrm{Bg}$ state photoelectron bands: (a) experimental spectrum; (b) theoretical spectrum taking into account the vibronic coupling between the three states obtained using the LVC model based on the parameters from the OVGF/cc-pVTZ calculations; (c) theoretical spectrum obtained using the same model as in (b), but without the vibronic coupling. This is equivalent to the Poisson spectra for the three states (see text for details). Individual transitions to vibronic states of $A_{g}$, $B_{u}$, and $B_{g}$ symmetry are shown in the spectra as green, red, and blue bars, respectively. The relative intensities of the spectra $(\mathrm{a}-\mathrm{c})$ were chosen in such a way that the height of the peak at the lowest binding energy in each spectrum is approximately equal to unity. The spectra were aligned so that the positions of these peaks, in each spectrum, coincide. 


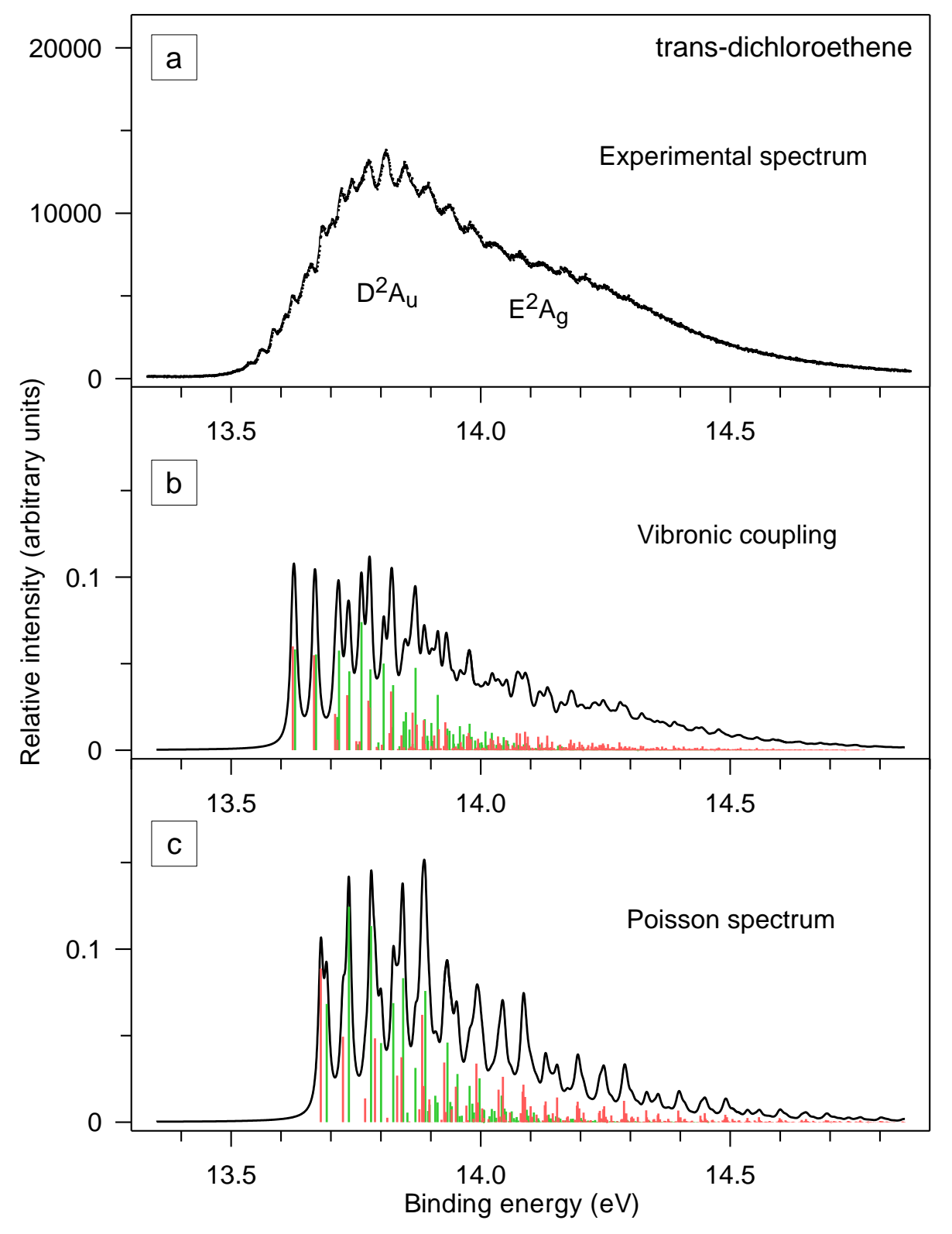

Figure 13

The $D^{2} A_{u}$ and $E{ }^{2} A_{g}$ state photoelectron band system: (a) experimental spectrum; (b) theoretical spectrum taking into account the vibronic coupling between the two states obtained using the LVC model based on the parameters from the OVGF/cc-pVTZ calculations; (c) theoretical spectrum obtained using the same model as in (b), but without the vibronic coupling. This is equivalent to the Poisson spectra for the two states (see text for details). Individual transitions to vibronic states of $A_{u}$ and $A_{g}$ symmetry are shown in the spectra as green and red bars, respectively. 


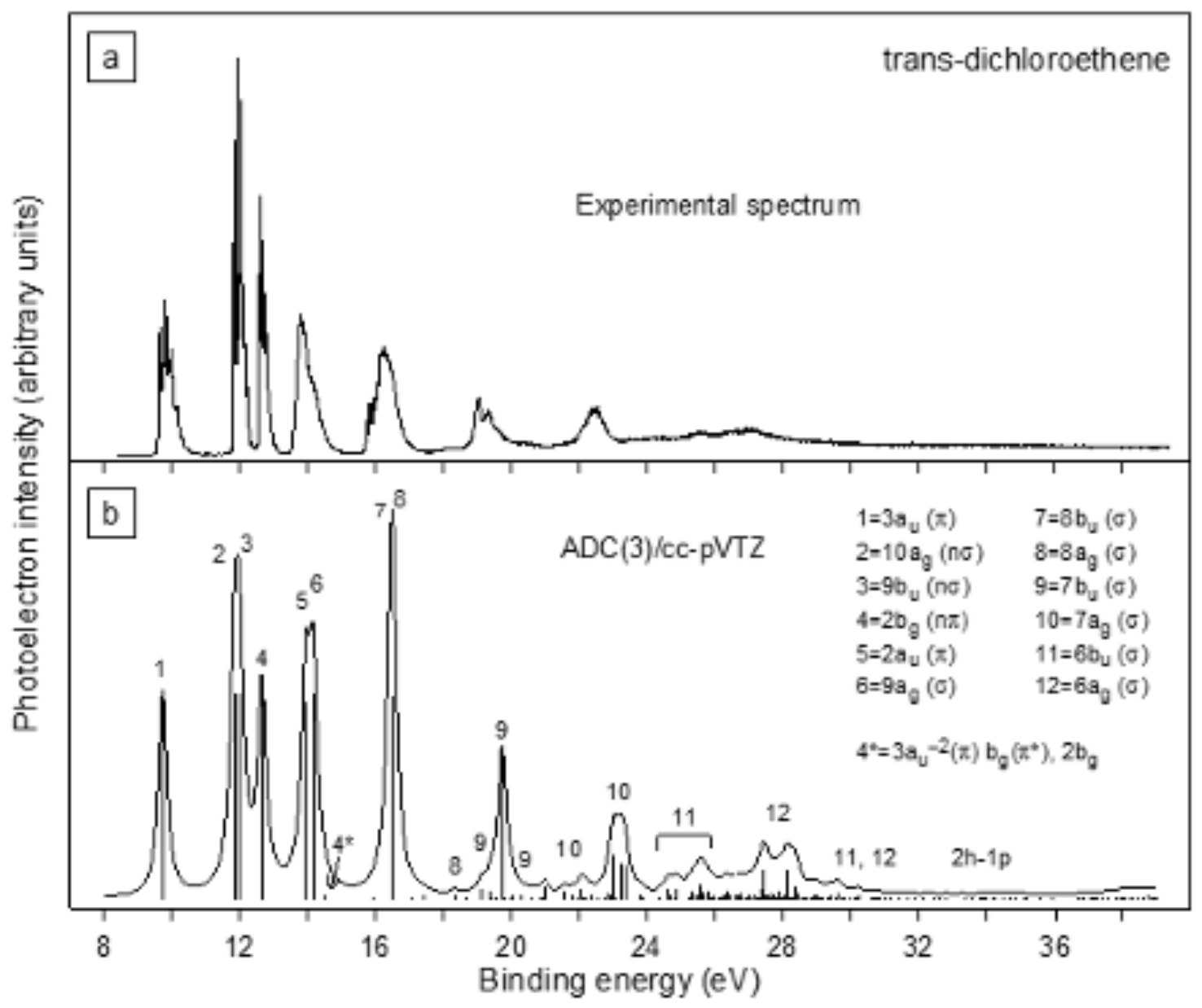




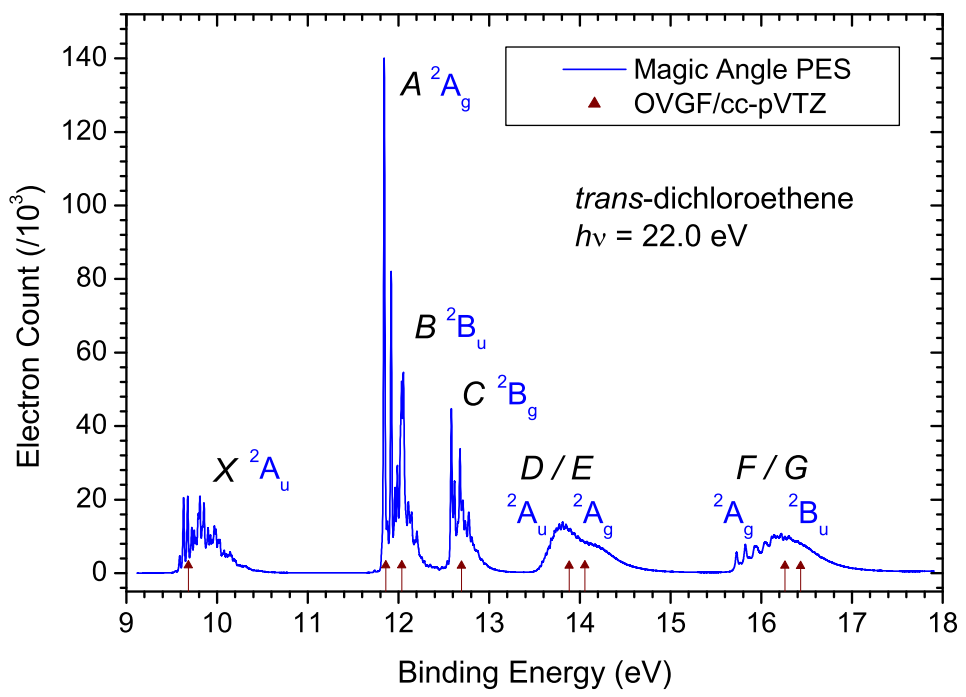


G

\section{$F$}

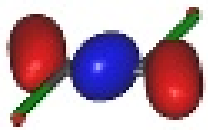

8ey

E

C
$9 \mathrm{a}_{9}$

D

B

$2 \mathrm{~b}_{9}$

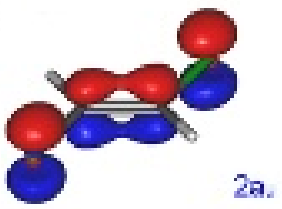

Etsu

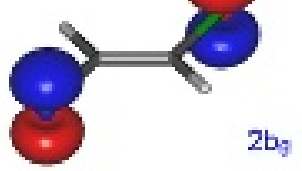

A

$x$

$10 \mathrm{~s}_{9}$

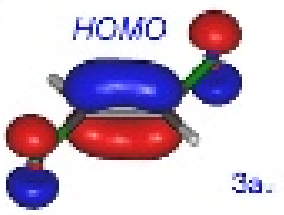




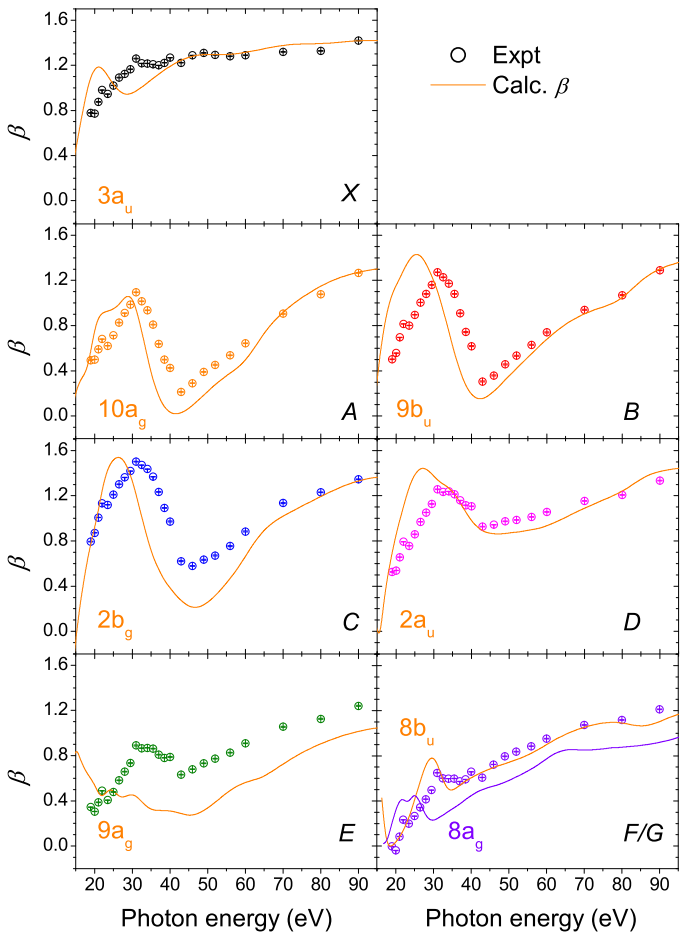




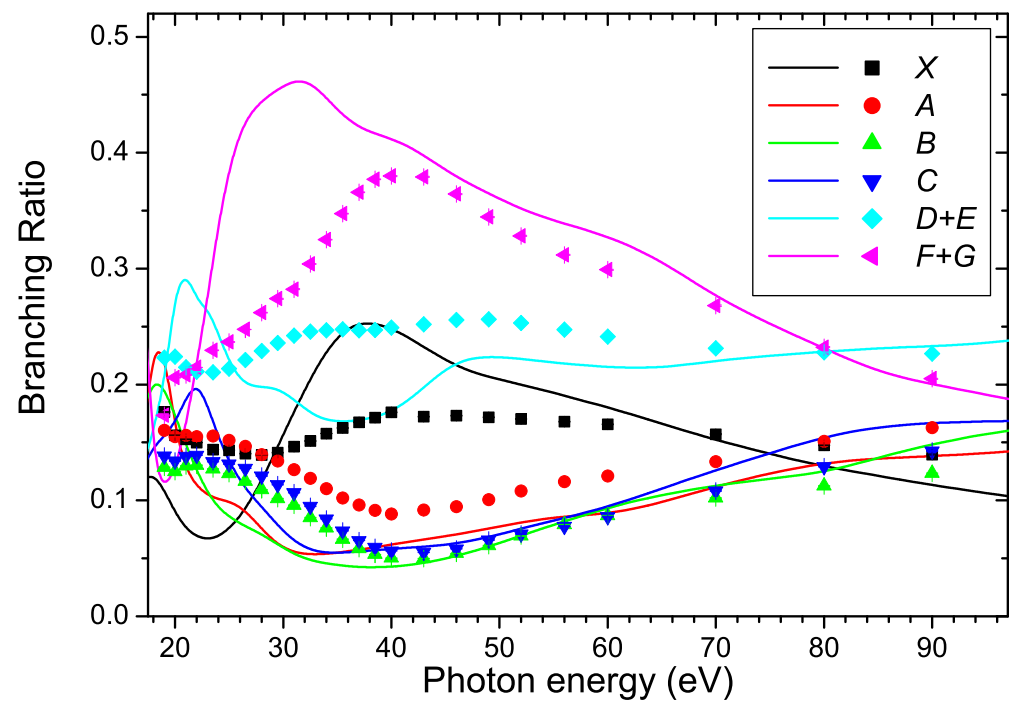




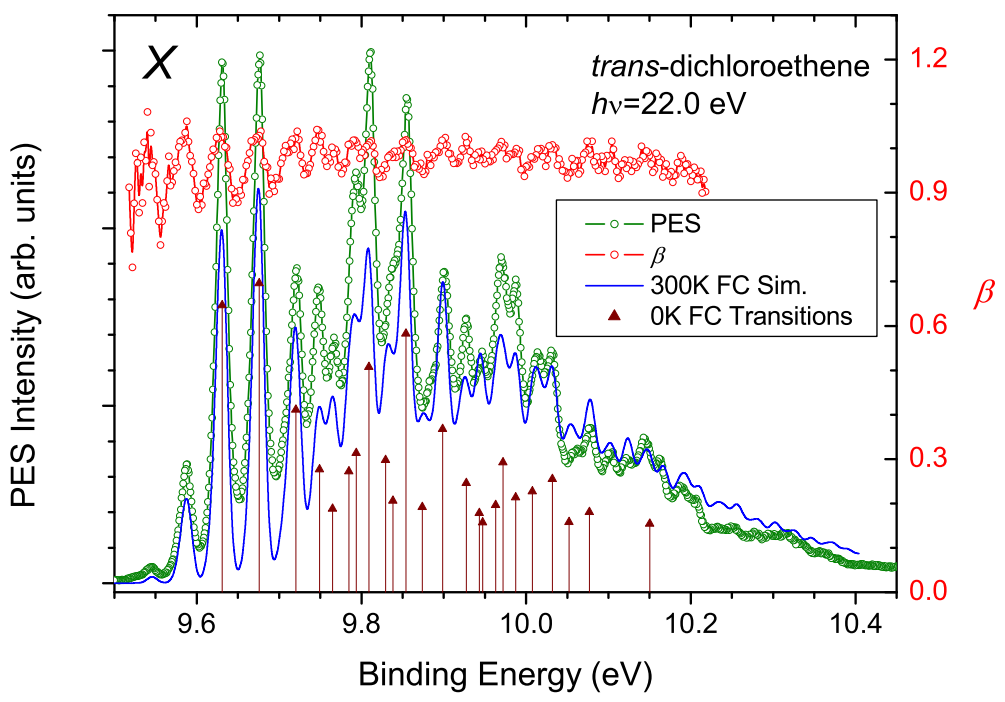




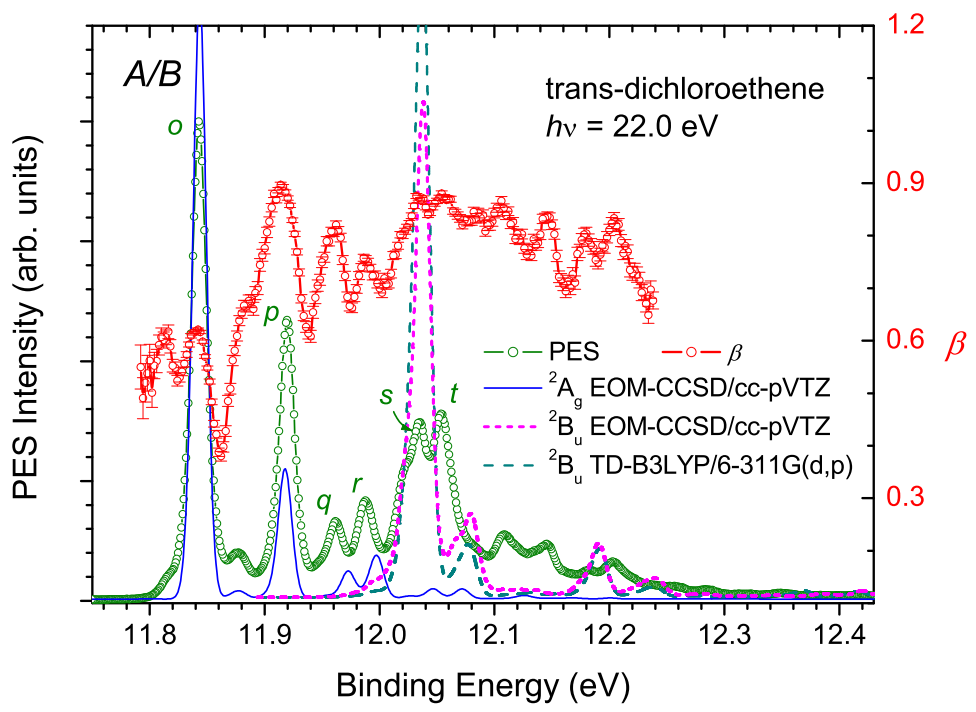




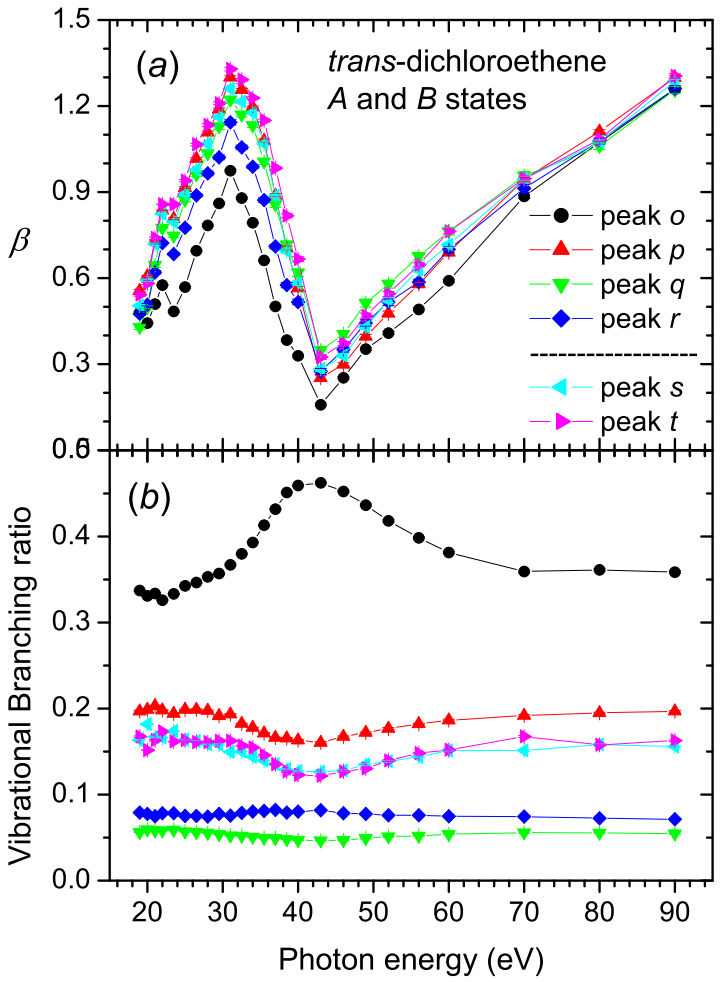




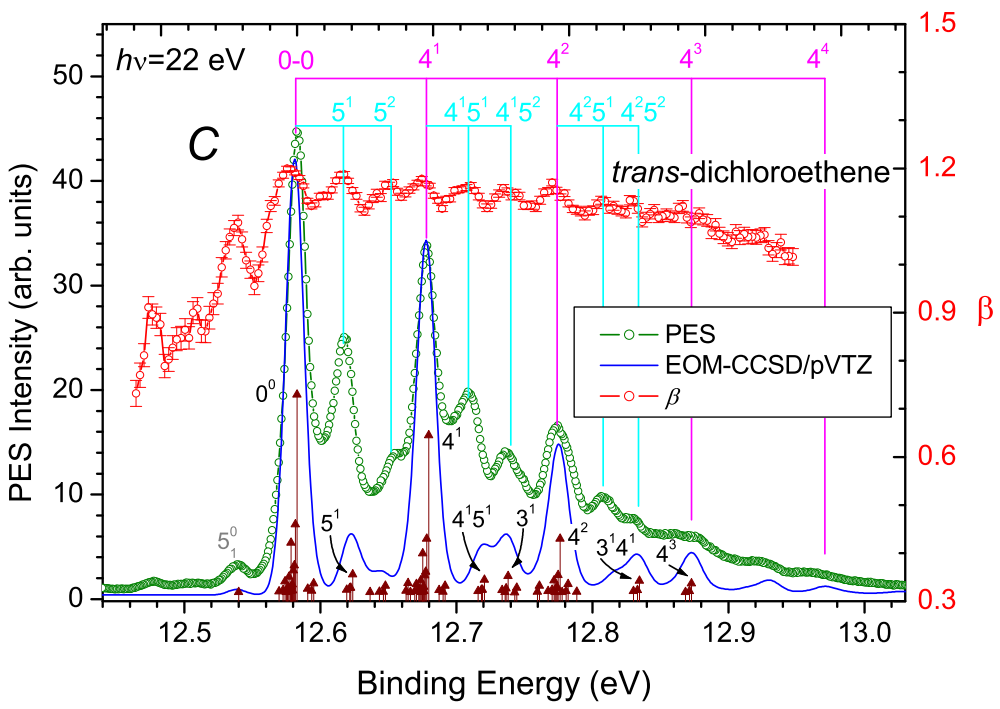




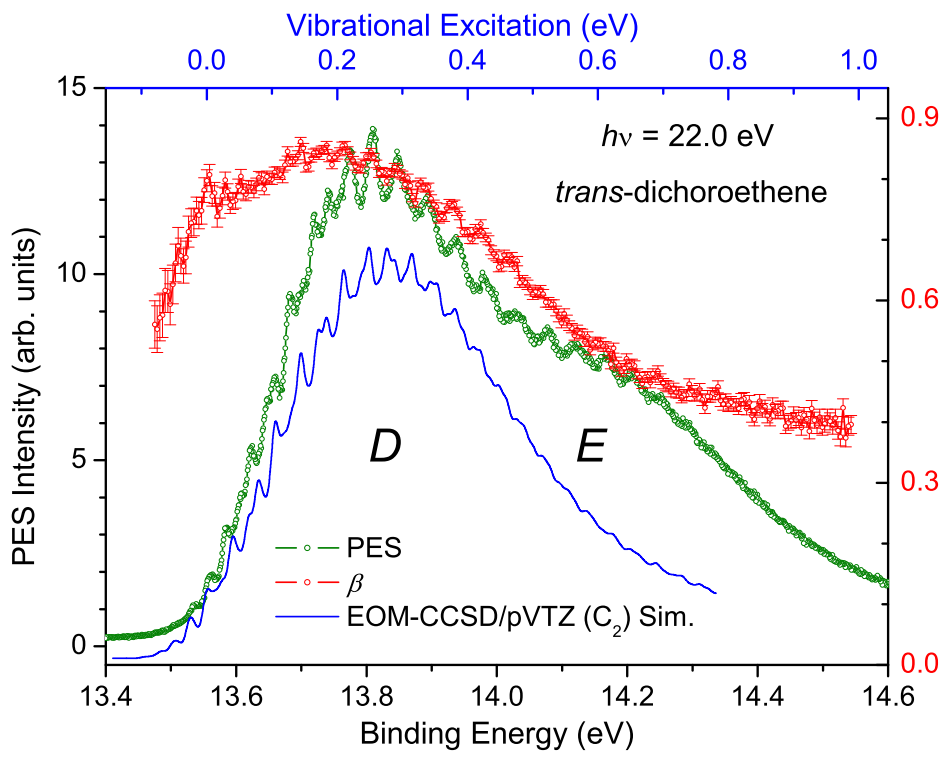




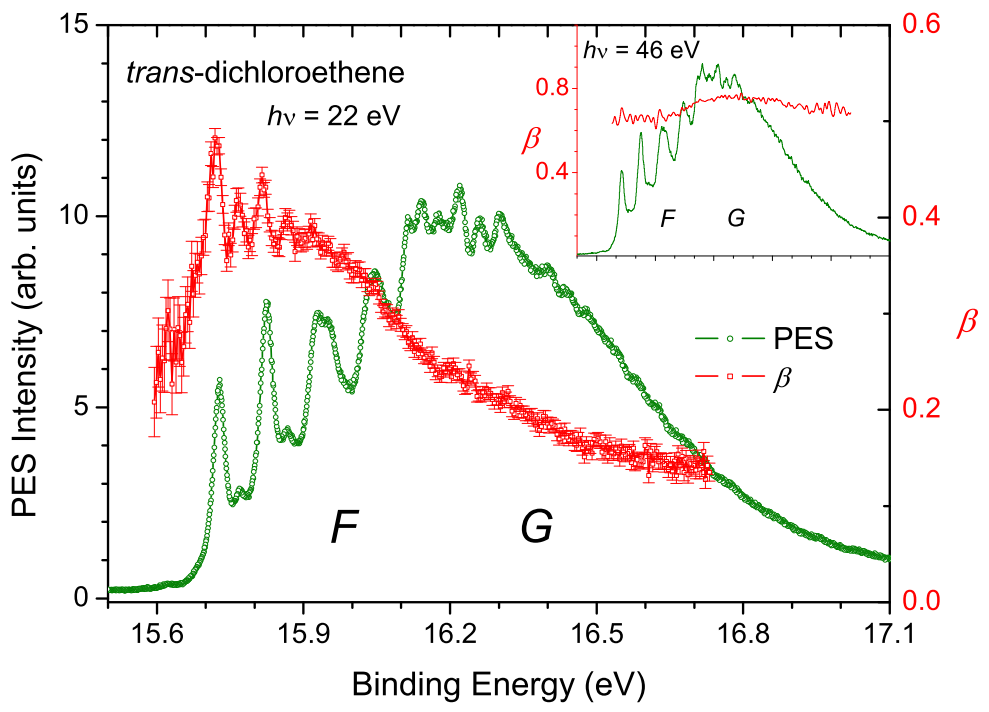




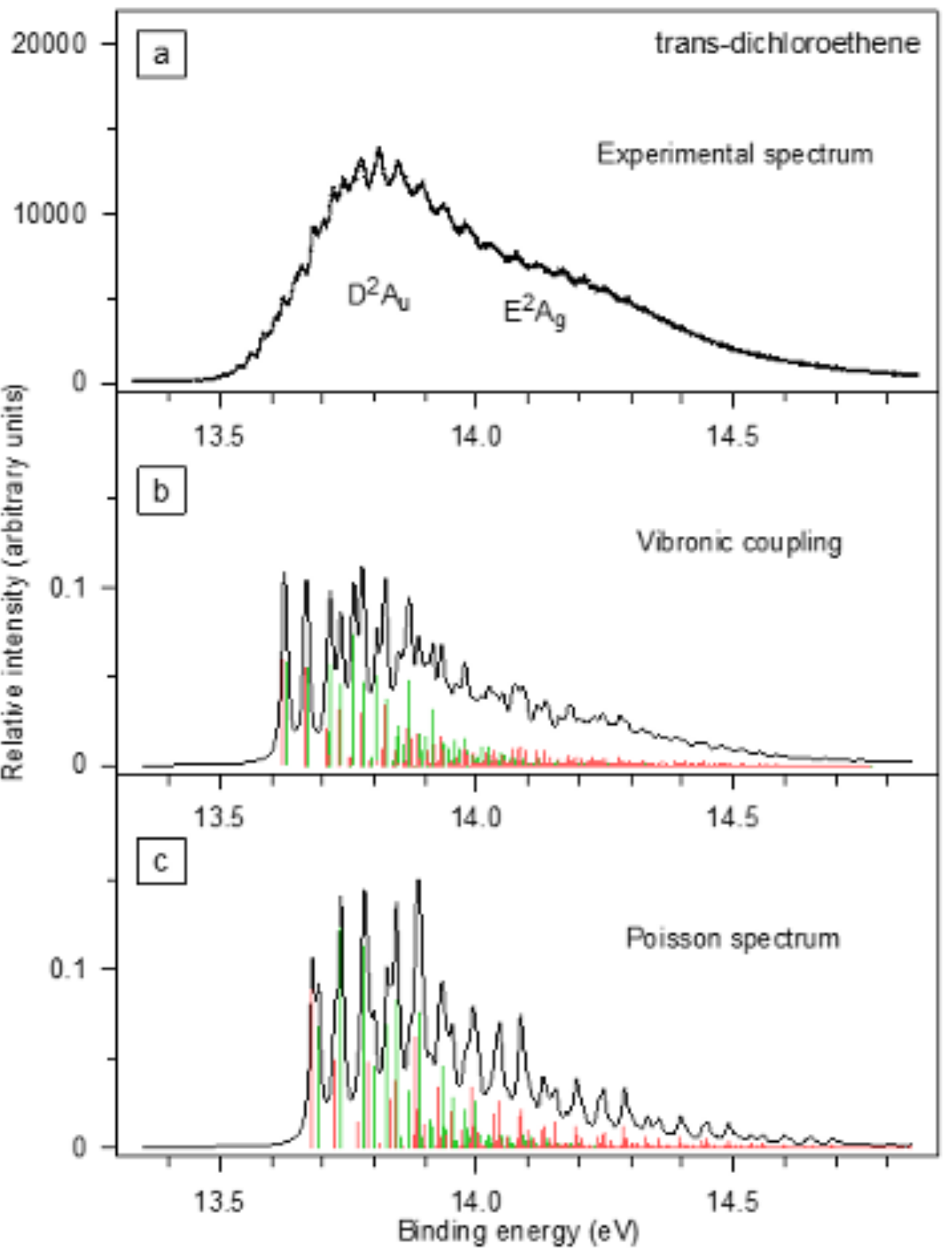

